\title{
Rursus
}

Russus

Poiétique, réception et réécriture des textes antiques

$1 \mid 2006$

Le modèle animal (I)

\section{La physiognomonie antique et le langage animal du corps}

Arnaud Zucker

\section{OpenEdition}

Journals

Édition électronique

URL : http://journals.openedition.org/rursus/58

DOI : 10.4000/rursus.58

ISSN : 1951-669X

Éditeur

Université Nice-Sophia Antipolis

Référence électronique

Arnaud Zucker, « La physiognomonie antique et le langage animal du corps », Rursus [En ligne],

1 | 2006, mis en ligne le 09 juillet 2006, consulté le 19 avril 2019. URL : http://journals.openedition.org/ rursus/58; DOl : 10.4000/rursus.58

Ce document a été généré automatiquement le 19 avril 2019

Rursus 


\title{
La physiognomonie antique et le langage animal du corps
}

\author{
Arnaud Zucker
}

\section{Introduction}

1 La physiognomonie, avant que ses avatars modernes ne la travestissent en pseudo-science (Dumont 1984, Laurand 2005), est une herméneutique antique dont les principaux représentants, s'ils sont sûrs de ses fondements, hésitent sur son statut épistémologique et laissent toujours au praticien droit de regard et dernier mot. Cette $\tau \dot{\varepsilon} \chi v \eta$ s'appuie en partie, mais profondément, sur l'observation zoologique qui lui fournit des repères, sinon des modèles, pour accéder au psychisme humain et à sa logique. Comme elle postule une intimité physique radicale de l'homme et de l'animal, elle alimente, du même coup, le soupçon d'anthropomorphisme qui pèse sur tout discours commun qui, hors du domaine strictement biologique, prétend ramener l'un et l'autre à la même échelle. S'agissant d'un $\lambda$ óyos dans lequel le corps animal apparaît en quelque sorte comme le révélateur, voire le signifié moral du corps de l'homme, ce handicap idéologique risque d'être rédhibitoire, au point que ceux-là même qui s'intéressent à la rationalité de ce discours trahissent leur gêne (Rodler 2000)ํ.

2 Il est ainsi doublement utile, pour réduire un malentendu partagé par ceux qu'elle attire et ceux qu'elle repousse, d'étudier la physiognomonie sous l'angle le plus négligé et le plus sensible de son discours, qui est le point d'ancrage de l'analogie qu'elle postule entre l'homme et les bêtes : l'analyse du corps. Dans l'étude de la zoologie physiognomonique on fait d'ordinaire et presque spontanément porter l'accent sur la psychologie (projective) des bêtes, alors que le point de passage privilégié et premier qu'elle pose entre l'homme et l'animal, c'est le corps. Après avoir rappelé les principes directeurs de la physiognomonie je m'attacherai à analyser dans les traités antiques la nature du bestiaire et la fonction des modèles animaux à travers l'étude des types et des équations morphologiques. Ce parti pris du corps conduira à réinterpréter l'approche zoologique dans les Physiognomonica aristotéliciens comme une extension de la méthode d'anatomie 
comparée qui est développée dans le corpus biologique, et à préciser la valeur paradigmatique du corps animal.

\section{Les bases de la physiognomonie}

\section{1. corpus}

Le corpus des traités physiognomoniques antiques a été rassemblé par Richard Foerster (1893) et aucune découverte ne l'a depuis lors substantiellement enrichi ${ }^{2}$. Il compte principalement (a) un traité péripatéticien $[43 \mathrm{p} .]^{3}$, dit du Pseudo-Aristote $\left(\mathrm{IV}^{\mathrm{e}}-\mathrm{III} \mathrm{I}^{\mathrm{e}} \mathrm{av}\right.$. J.C.), sans doute constitué de deux parties (\$1-3 puis §4-6), l'une et l'autre lacunaires (Degkwitz 1988 : 6-7 ; Foerster 1893 : XVIII), composées et combinées à la même époque, voire par un seul auteur (Vogt 1999: 192), ou dont la première partie serait de peu antérieure à la seconde (Degkwitz $1988: 5)^{4}$; (b) un long traité [97 p.] du sophiste Polémon (II ${ }^{\mathrm{e}}$ ap. J.C.) parvenu sous la forme d'une traduction arabe, traduite en latin par $\mathrm{H}$. Schmoelder, au XIXe ; (c) un traité [130 p.] du médecin Adamantios (IVe ap. J.C.) qui constitue une paraphrase de Polémon; (d) un anonyme latin [142 p.], attribué autrefois à Apulée, auteur attesté d'un traité de ce genre, dont une étude de langue (André 1981 :32) fixe la rédaction au IV ap. J.C.; enfin (e) un traité byzantin (Xe ap. J.C.), dit du PseudoPolémon, qui est un épitomé d'Adamantios 5 .

\section{2. principe et définition}

4 Une des caractéristiques de la littérature physiognomonique antique est de se présenter comme un discours à la fois théorique, historique et critique sur la technique qu'elle développe. Ainsi se trouvent rappelés dans chacun des traités qui nous sont parvenus, depuis le premier attribué à Aristote, l'objet, les principes et la méthode de cette discipline. "La physiognomonie, comme son nom l'indique, traite des manifestations physiques des dispositions de l'esprit et des caractères acquis venant modifier les signes qui font l'objet d'une analyse physiognomonique. [...] On appréhende le caractère à partir des mouvements, des poses, des couleurs, des expressions du visage, des cheveux, de la finesse de la peau, de la voix, de la chair, des parties du corps ainsi que de la forme d'ensemble du corps " (Arist., Phgn. 806 a 22-33) ${ }^{6}$. Cette discipline est donc une technique d'interprétation qui permet de remonter du corps à l'esprit et postule, fondamentalement, à la fois une dualité et une complicité psycho-somatique.

5 La corrélation intime et l'influence réciproque de la partie psychique et de la partie somatique en l'homme, et dans les animaux en général, est l'ỏ $\rho x \eta ́$ aussi bien logique que théorique du discours physiognomonique: « les dispositions mentales se règlent sur la

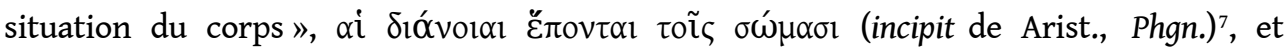

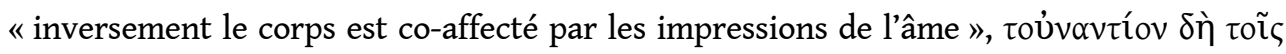

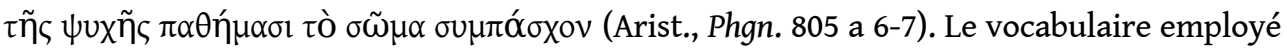
pour désigner les deux instances en sym-pathie ${ }^{8}$ varie peu d'un traité à l'autre et

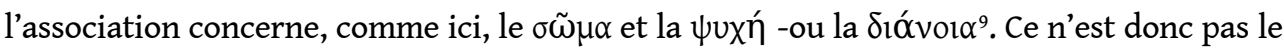
caractère, au sens simplement affectif et moral de трó́тоৎ que vise la physiognomonie mais l'ensemble des dispositions mentales et intérieures. Son enjeu est la psychologie au

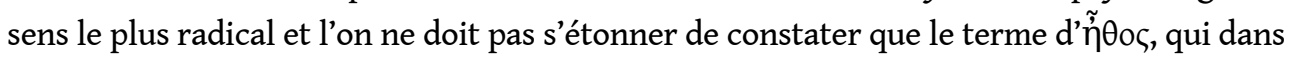
les grands textes zoologiques ne cerne qu'un aspect limité et apparent de la $\psi v x \eta ́$, ait 
dans les Physiognomonica aristotéliciens un sens strictement somatique et superficiel et désigne les "expressions d'humeur ", essentiellement faciales, et non le tempérament à découvriri ${ }^{10}$.

6 Cette ambition de reconstituer le portrait psychique complet d'un homme, à partir des données visibles qu'il expose inévitablement, repose sur un credo scientifique fondamental et général exprimé par la célèbre formule anaxagoréenne ǒ $\psi 1 \varsigma \tau \tilde{\omega} v \dot{\alpha} \delta \eta ́ \lambda \omega v$

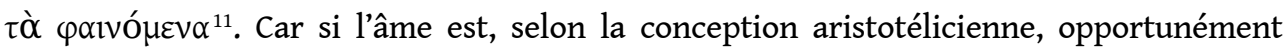
rappelée par Galien dans son traité sur la solidarité de l'esprit et du corps, l' $\varepsilon \tilde{i} \delta$ oc du corps

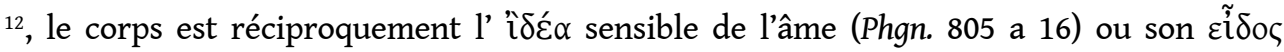
(Adamant. 1, 4). La physis, objet de la connaissance de la physiognomonie, est donc d'abord la nature intime, intus et in cute, des individus qu'informe et révèle leur apparence ${ }^{13}$. Mais, plus profondément, la connaissance de la nature est la connaissance et la compréhension de l'articulation psychosomatique ${ }^{14}$, et le sens de la sympathie de l'âme et du corps réside philosophiquement -et anthropologiquement- moins dans l'interprétation des signes que dans l'appréhension de l'unité du signe (psychophysique). Cette unité, qui constitue donc à la fois le postulatum et le demonstrandum de la démarche physiognomonique, trouve dans la langue une complicité profonde, en particulier dans la communauté du vocabulaire et

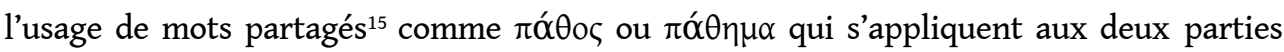
constitutives de l'être animé et peuvent exprimer un affect passager aussi bien qu'un état durable (Bonitz 1867).

\section{3. axiomes}

$7 \quad$ L'interdépendance du $\sigma \tilde{\omega} \mu \alpha$ et de la $\psi v x \eta ́$ qui constitue le fondement épistémologique de la physiognomonie ${ }^{16} \mathrm{~s}^{\prime}$ exprime à travers trois axiomes: (1) Les dispositions mentales ne sont pas autonomes et insensibles aux mouvements du corps. (2) Le corps réciproquement est affecté par les affects de l'âme. (3) Un trait mental est corrélé à un trait physique ${ }^{17}$. Les deux premiers sont explicites (Phgn. 805 a 1-7 et 808 b 12-15) mais non le troisième qui est pourtant le seul énoncé caractéristique de la discipline, et qui pose de nombreux problèmes théoriques, en particulier de nature sémiologique (Laurand 2005). Si néanmoins il faut le considérer comme un axiome c'est parce que toutes les équations psycho-physiques particulières supposent cette règle et en découlent.

\section{4. syllogismes et méthodes}

D’après le début des deux parties du traité d'Aristote (\$1 et \$4) on peut reconstituer le syllogisme général de la physiognomonie : (a) les affections de l'âme et celles du corps s'influencent réciproquement; (b) or il existe dans les êtres (et la vie affective) une correspondance constante entre le physique et le mental ; (c) donc l'apparence physique

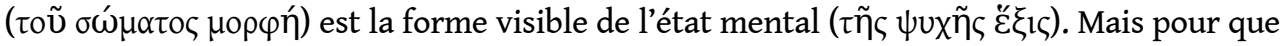
le physiognomoniste puisse utiliser les signes, autrement dit apparier correctement chez un individu les traits physiques aux traits mentaux correspondants il faut qu'il aie au préalable identifié ces signes en en percevant, pour ainsi dire, les deux faces simultanément. En effet, pour éviter d'être une généralisation empirique et inductive et se donner, au contraire, la forme d'un raisonnement déductif, la physiognomonie doit avoir accès à un domaine de référence où les signes psychosomatiques se présentent clairement, et surtout dans leur plénitude. Trois régimes du corps paraissent sur ce point 
donner satisfaction et offrir des signes immédiatement lisibles et évidents : le corps ému, le corps étranger et le corps animal. Ils déterminent la tripartition traditionnelle de la physiognomonie en trois méthodes, selon qu'elle s'appuie sur une sémiologie pathognomique (les expressions communes des émotions), ethnologique (les traits physiques typiques des peuples) ou zoologique (les particularités anatomiques des animaux). Les trois registres de référence jouent dans le discours à peu près le même rôle (bien que leur extension et leur potentiel ne soit pas identiques), et l'on pourrait pour les autres registres proposer une formulation semblable à celle que nous proposons $d u$ syllogisme de la méthode zoologique ${ }^{18}:\left(a^{\prime}\right)$ en chaque espèce animale l'anatomie correspond aux propriétés psychiques ; ( $\left.b^{\prime}\right)$ or les traits caractéristiques de ces anatomies se retrouvent chez l'homme; $\left(c^{\prime}\right)$ donc l'homme qui possède les mêmes traits a des propriétés psychiques analogues.

Il apparaît donc que pour pratiquer convenablement la méthode zoologique il faut donc que le physiognomoniste soit zoologue, c'est-à-dire connaisse les parties physiques et le caractère des animaux ${ }^{19}$; et, de plus, que nécessairement la physiognomonie de l'animal précède et conditionne celle de l'homme ${ }^{20}$. Aux yeux de certains l'invisible seul, chez l'animal, pose problème, et il suffit au physiognomoniste de s'informer sur la valeur psychique des bêtes ${ }^{21}$; mais le traité aristotélicien, plus lucide, insiste sur le rôle des

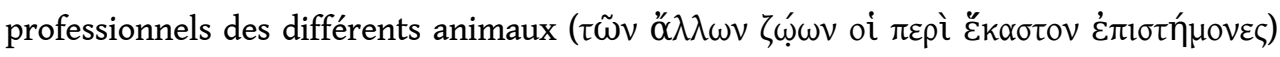
sur le savoir spécialisé desquels le physiognomoniste qui étudie l'homme selon la méthode zoologique doit s'appuyer, car ils sont capables de tirer des conclusions

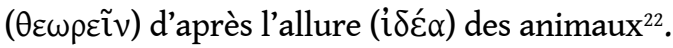

\section{5. problématiques classiques et question du corps}

La physiognomonie antique, expression d'une conception souvent diffuse et peu formalisée mais culturellement irradiante, a été étudiée selon de nombreuses perspectives dont j'indique rapidement les principaux centres d'intérêt, avant de m'engager dans l'analyse du discours anatomique proprement dit: [1] l'influence littéraire et iconographique de ce discours (Evans 1969; Kiilerich 1988; Vogt 1999, 45 sq.) ; [2] le statut épistémologique de la physiognomonie, entre médecine et philosophie (Lombardi 1999), et sa présence dans ces deux traditions (théorie des climats, écologie, sympathie et antipathie:Evans 1969, 17-28; Tsouna 1998); [3] le développement historique de l'antiquité à nos jours de cette discipline (Baltrusaitis 1995, Caro Baroja 1988, Lapini 1992, Pack 1974, Schmidt 1941), en particulier au Moyen-Âge (Agrimi 1993, 1996) et à l'époque moderne (Dumont 1984, Rodler 2000) ; [4] l'analyse des différentes méthodes de la physiognomonie (MacC. Armstrong 1958, Laurand 2005) ; [5] la sémiologie physiognomonique (Laurand 2006, Manetti 1987, Zucker 2006) ${ }^{23}$; et [6] le rapport de la physiognomonie avec la caractérologie (Théophraste) et la psychologie des animaux (Dierauer 1977, Lloyd 1983, Lombardi 1999). Je vais m'attacher à un aspect moins développé dans la littérature critique et qui consiste dans le rapport précis établi entre le corps animal et le corps humain. Si la nature humaine est connaissable par le biais de signes externes que l'animal offre en clair, la similitude entre l'homme et l'animal ne peut être simplement psychologique et globale, elle doit être aussi somatique et analytique, et les équations précises que propose la physiognomonie constituent des indices d'une représentation particulière de la relation entre l'un et l'autre. 


\section{La zootypie}

\section{1. le bestiaire du physiognomoniste}

11 Le bestiaire des traités de physiognomonie privilégie les quadrupèdes, mais intègre aussi des animaux morphologiquement très distants. Voici la liste des animaux mentionnés dans les quatre principaux traités antiques ${ }^{24}$ :

- le bestiaire d'Aristote (20) : [quadrupèdes] lion, chien, âne, chèvre, cochon et sanglier, singe, guépard, bœuf, cheval, mouton, renard, loup, lièvre, cerf, chat ; [oiseaux] aigle, corbeau, coq, faucon; [amphibiens et reptiles] grenouille ; [termes généraux] rapaces, fauves, palmipèdes, oiseaux ;

- le bestiaire de Polémon $(10 / 85)^{25}$ : [quadrupèdes] lion, chien, cochon, singe, renard, âne, chèvre, guépard, loup cervier, lynx, hyène, loup, ours, chacal, thos, loir, taupe, porc-épic, hérisson (erinaceus parvus), varan, éléphant, rhinocéros, buffle, boeuf, chameau, girafe, bœuf sauvage, cerf, antilope (ibex), gazelle (dorcas), mouton, chevreuil, cheval, mulet, onagre, zibeline, hippopotame ; chauve-souris ; [amphibiens et reptiles] crocodile, serpent, tortue grenouille, lézard ; [oiseaux] aigle, faucon, épervier, sacre, vautour, percnoptère, milan, corbeau, corneille, pie grièche, pie, oie, canarde, grue, porphnos, autruche, paon, poule, coq, pintade, perdrix, colombe, caille, étourneau, passereau, pinson, hirondelle (appus), huppe, ganga cata (pterocles alchata); [poissons] squale, requin carcharias anguille; [autres animaux marins] langouste, baleine, dauphin ; [arthropodes] sauterelle, abeille, guêpe, mouche, scorpion, fourmi ; [termes généraux] oiseaux, bêtes sauvages, bêtes domestiques, poisson;

- le bestiaire d'Adamantios (12/19) : [quadrupèdes] lion, guépard, chien, cochon et sanglier, bœuf, cheval, mouton, âne, chèvre, singe, loup, ours, ichneumon, renard ; [oiseaux] perdrix ; [amphibiens et reptiles] dragon, vipère ; [autres animaux marins] monstre marin ; [termes généraux] oiseaux, serpent ;

- le bestiaire de l'Anonyme Latin (19/27) : lion, bœuf, chien, cerf, cheval, âne, singe, cochon, chèvre, loup, ours, guépard, ichneumon, renard ; [oiseaux] paon, chouette, oie, corbeau, coq, aigle, perdrix, épervier, paon, pie ; [amphibiens et reptiles] grenouille, tortue ; [termes généraux] serpent.

Le bestiaire est donc instable et on ne compte que six zoonymes communs à toute la tradition: le lion, le chien, l'âne, la chèvre, le cochon et le sanglier, le singe. Il est également réduit. La liste considérable donnée pour Polémon est trompeuse, car la plupart des animaux ne sont pas exploités physiognomoniquement, mais figurent dans une liste, déconnectée de toute relation au corps de l'homme, qui attribue succinctement à chaque animal un lot de qualificatifs (de deux à sept) moraux et psychologiques, du type: "dauphin: joueur, concupiscent, inoffensif; requin: fourbe, dissolu, perfide, fuyant, audacieux... $»^{26}$. L'Anonyme latin, en revanche, reprenant à Polémon la présentation sous forme d'inventaire, offre dans une formule complémentaire un répertoire de quatorze portraits physiognomoniques, dans lesquels il décrit successivement l'animus d'un animal, puis le corpus et l'animus de l'homme qui lui correspond (homines ad hujus animalis speciem referuntur) ${ }^{27}$. Il faut donc distinguer le bestiaire actif du bestiaire passif. L'actif, constitué des animaux effectivement utilisés comme références éclairantes pour une lecture caractérologique humaine, est très réduit (de 10 à 20 notions), si l'on s'en tient aux espèces, tout en sachant que dans les espèces à dimorphisme sexuel important les deux sexes sont mentionnés isolément. Ce capital 
morphologique, constitué majoritairement par des animaux proches (bétail, quadrupèdes sauvages et oiseaux) offre une réserve suffisante de variables physiques ${ }^{28}$.

\section{2. la constitution de types}

13 Les animaux sélectionnés sont, au niveau symbolique, culturel et classificatoire, non seulement des animaux majeurs, mais également des sortes de prototypes ${ }^{29}$. Dotés d'une valeur paradigmatique ils catalysent en eux un grand nombre de représentations et de marques affectives jusqu'à former éventuellement des paires de contraires, autrement dit des notions pour figurer des concepts bipolaires essentiels à l'appréhension des différences psychiques entre les êtres: féminin/masculin, courageux/lâche, simple/ perfide, etc. «Le lion et le sanglier répondent au type masculin, le guépard, le cerf et le lièvre au féminin; l'aigle et l'épervier au type masculin, le paon, la perdrix et la pie au féminin » leo, aper ad masculinum genus referuntur, pardus, cervus, lepus ad femininum, aquila, accipiter ad masculinum genus, pavus, perdix, pica ad femininum ${ }^{30}$. Adamantios (2.2) propose ainsi une série de trois paires de modèles animaux, illustrant l'opposition entre les deux caractères sexuels et fonctionnant dans trois registres classificatoires différents : lion vs guépard, aigle vs perdrix, dragon $v s$ vipère.

Ces animaux de référence sont donc des notions pleines et en même temps construites. Tant dans leur description physique que dans leur portrait psychique ils apparaissent comme des idéalisations, ou, si l'on veut (mais c'est à peine autre chose) comme des idéaux. La nature radicale -voire caricaturale- du complexe psychique attribué aux animaux dans la littérature psychologique, même lorsqu'elle ne relève pas strictement d'une tradition populaire mais s'intègre à une perspective zoologique exigeante, est une donnée régulière et bien connue. Aristote, au début de l'Histoire des animaux, évoquant les

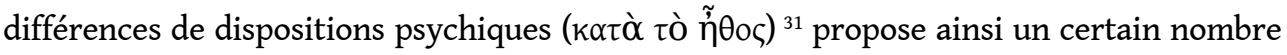
de configurations typées : "Les uns sont doux, nonchalants, sans obstination, comme le bœuf, d'autres sont pleins d'ardeur, obstinés, stupides, comme le sanglier, d'autres sont prudents et timides, comme le cerf, le lièvre, d'autres sont vils et perfides, comme les serpents, d'autres sont nobles, braves et généreux, comme le lion, d'autres sont racés, féroces et perfides, comme le loup, <...> d'autres sont rusés et méchants, comme le renard ; d'autres ont du cœur, sont capables d'attachement, caressants, comme le chien ; d'autres sont doux et faciles à apprivoiser, comme l'éléphant; d'autres son pudiques et toujours sur leurs gardes, comme l'oie; d'autres sont jaloux et orgueilleux, comme le paon ${ }^{32}$. Mais on peut s'étonner que ce schématisme affecte également dans la physiognomonie la représentation morphologique des animaux et que le portrait qui en est fait relève davantage de l'idéotype que du phénotype. Pourtant, si l'on considère les données descriptives des traités, il apparaît qu'à toute notion animale correspond une figure homogène et figée, même lorsque la littérature zoologique reconnaît au genre nommé une multitude de différences intragénériques, comme pour le lion, le cheval ou le chien $^{33}$. L'ellipse des différences raciales permet la constitution d'un type du chien à la fois fonctionnel et fictionnel. La neutralisation des différences spécifiques ou variétales, qui indique un appauvrissement volontaire du matériel zoologique, s'accompagne d'une négation de la plastique du corps animal, qui se trouve figé dans une pose unique.

Cette simplification anatomique est à la fois le corollaire du schématisme psychique ${ }^{34}$ et la condition même du fonctionnement de la figure animale comme signe physiognomonique. La logique du corps est différente selon homme et selon l'animal : le 
corps animal doit être impassible car il est tout entier expression d'une physis; au contraire l'homme a une mobilité, des mimiques différentielles, un tropos individuel. Si la nature (psycho-somatique) de l'animal est un intermédiaire éclairant pour saisir celle de l'homme, c'est dans la mesure où le signe animal se donne d'emblée et n'est pas parasité par des accidents ou des écarts individuels. Le signe animal est idéal et parfait car chez l'animal les éléments du corps (sélectionnés) sont des lettres physiognomiques qui n'ont pas besoin d'être interprétées, puisqu'elles font en quelque sorte "corps" avec leur porteur et que les animaux offrent une continuité de sens entre $\sigma \tilde{\omega} \mu \alpha$ (morphologie), $\tilde{\hat{\eta}} \theta$ os (expressions du corps) et $\psi u x \eta ́$ (dispositions psychiques). Les animaux ont, bienheureusement, le physique qui leur convient: «jamais aucun animal, dit Aristote, n'est tel qu'il a l'apparence physique d'un animal et les dispositions psychiques d'un autre; le donné psychique et le donné somatique correspondent systématiquement à un seul et même animal, et il existe ainsi une détermination automatique d'un certain donné

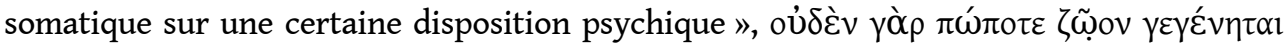

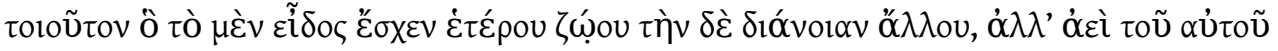

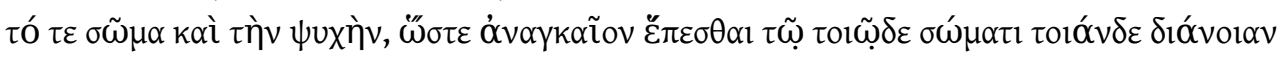
${ }^{35}$. Cette mise en scène de la nature animale illustre bien la tautologie ou plutôt la redondance qu'elle constitue ${ }^{36}$. La simplicité de la structure animale est une élaboration stratégique ${ }^{37}$.

\section{3. le statut idéal des cinq critères formels du physiognomoniste}

Mais ce processus d'abstraction affecte tous les types qui sont amenés à jouer, comme les figures animales, le rôle de critères dans le discours physiognomonique. On a signalé plus haut l'importance de la différenciation sexuelle (masculin/féminin) pour apprécier les différentes dispositions psychiques. Dans les Physiognomonica elle est présentée comme une différence majeure qui oriente toute l'interprétation psychique et partage physiquement l'animalité -et l'humanité- en deux: "Il faut diviser l'animalité en deux

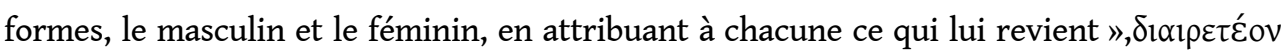

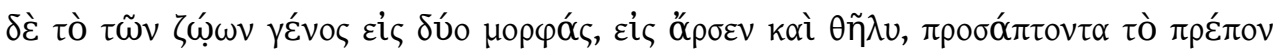

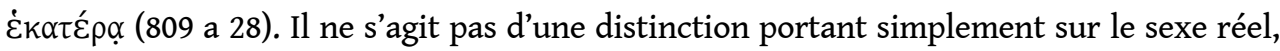
mais d'un clivage qui se déporte sur le sexe psychique et conséquemment psychosomatique, pour désigner deux façons d'être. Les représentants paradigmatiques de ces deux options sont le lion (masculin) et le guépard (féminin), deux espèces dont l'anatomie est à la suite longuement décrite. Leur opposition se manifeste en particulier

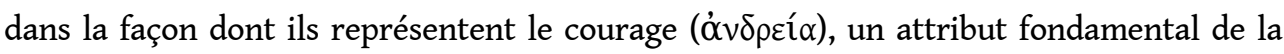
$\psi v \times \eta^{38}$, qu'ils incarnent chacun d'une façon exemplaire $(\tau \varepsilon \lambda \varepsilon \omega ́ \tau \tau \alpha \tau \alpha)^{39}$ et inverse,

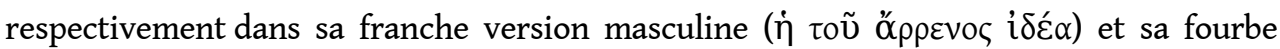

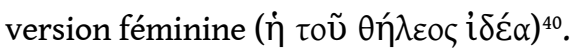

Le traité joue en fait sur un double niveau et use d'un double langage, car il n'exclut pas tout à fait la détermination sexuelle (tout comme il exploite l'opposition marquée par le

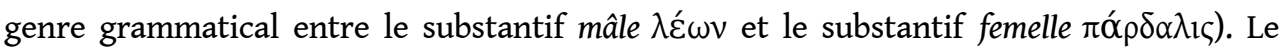
cinquième chapitre du traité péripatéticien est en effet entièrement consacré au développement successif des deux acceptions de la différence sexuelle: l'acception physique et littérale (qui reprend l'analyse de l'opposition sexuelle et de la supériorité du mâle esquissée au livre IX de HA en 608 a33- b 4), puis l'acception psychique et idéale, qui est spécifique à ce traité. Ce glissement s'effectue à travers la figure opportune du 
guépard, espèce chez laquelle, de manière exceptionnelle, la valeur est selon HA du côté de la femelle (sexuellement parlant) et non du mâle ${ }^{41}$, et qui se trouve, dans les Physiognomonica, chargée de porter les couleurs de la féminité même. L'Anonyme latin confirme cette mue du concept en physiognomonie dans son exégèse de la prima divisio atque discretio de la discipline, qui concerne le femininum et le masculinum genus (glosés

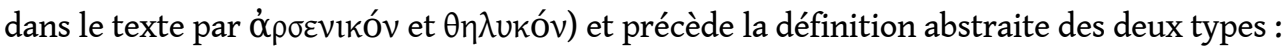
«ce ne doit pas être compris au sens où nous l'entendons de la distinction naturelle entre les sexes et les genres, mais en ce sens que, d'une façon générale, on trouve aussi un type masculin dans le féminin et un féminin dans le masculin », quod non ea ratione accipiendum est qua naturaliter sexus et genera discreta sunt, sed ut plerumque etiam in feminino masculinum genus et in masculino femininum deprehendatur (AnL 3).

Les critères invoqués alternativement par l'auteur de la deuxième partie des Physiognomonica à l'appui de chacune de ses équations sur le signifié psychique d'un trait physique ${ }^{42}$, et qui sont exploités ensuite dans les traités postérieurs, sont au nombre de cinq, et constituent tous des idéotypes. Outre le double critère du genre (masculin/ féminin) et les zootypes qui composent le bestiaire actif, ce sont les pathotypes, les

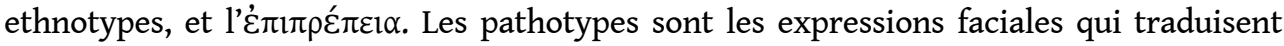
une émotion passagère (comme la peur ou la colère), un penchant (comme l'alcoolisme, la somnolence) ou une humeur (la plainte ou la tristesse) et offrent une clé pour l'interprétation des traits physiques que présentent, au repos, les visages. Les ethnotypes, davantage sollicités par les traités médicaux ${ }^{43}$, sont les portraits exagérés des peuples barbares. Le dernier critère, innovation de la deuxième partie du traité aristotélicien ${ }^{44}$, qui constitue le cœur spéculatif de la physiognomonie et qui en est aussi, selon l'Anonyme latin, la maxima pars (AnL 45), est représenté par un terme abstrus pour lesquels les traducteurs hésitent sans s'accorder ${ }^{45}$. Le mot غं $\pi ı \rho \varepsilon^{\prime} \pi \varepsilon ı \alpha$ est un terme rare, et peut-être délibérément vague, qui renvoie à une "convenance " et à une "harmonie " jamais explicitée, et qui doit être un idéal intuitif ${ }^{46}$. Ces cinq critères de l'évaluation

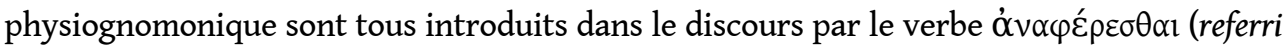

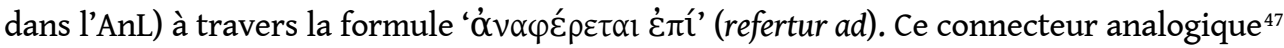
implique un mouvement de remontée vers un principe et un rappel (Bonitz $1870: 54 a$ ), qui semble indiquer un signifié plutôt qu'une image et pointe une origine plutôt qu'une cause $^{48}$. S'agissant du critère qui nous occupe, le renvoi à un animal consacre la figure zoologique typique dans un statut de patron formel pour l'individu analysé, -patron qui a au minimum une valeur analogique, mais peut intervenir comme une référence archétypique $^{49}$.

\section{L'anatomie comparée}

\subsection{I'analyse physique des animaux paradigmatiques}

Les types animaux fournissent donc, individuellement mais selon une procédure analogique commune, un critère pour évaluer dans l'individu humain son versant psychique. Cette fonction et le caractère idéal du type ne doivent pas occulter la pertinence zoologique et la précision anatomique des figures animales. Ainsi les deux animaux clés, le lion et le guépard, reçoivent un signalement très précis (Phgn. $809 \mathrm{~b}$ 14-810 a 8) et chacune des parties physiques isolées et qualifiées est susceptible de guider une équation et de servir de repère. Les parties notables -et donc humainement 
significatives- du lion sont : la gueule, le visage, la mâchoire inférieure, le nez, les yeux, les sourcils, le front, la tête, le cou, les clavicules, les épaules, la poitrine, le dos, les flancs, la croupe, les jambes ${ }^{50}$; celles du guépard sont : le visage, la gueule, le front, les yeux, le cou, la poitrine, le dos, la croupe, les cuisses, les flancs, le ventre, les jambes ${ }^{51}$. Ces détails

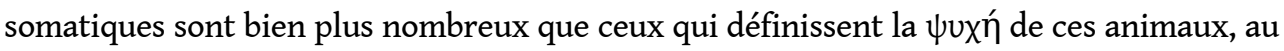
point que ces descriptions anatomiques sont, à notre connaissance, les plus complètes que l'on trouve pour le lion et le guépard dans la littérature grecque. Ils permettent aussi d'exclure, pour le second, l'identification traditionnelle et reprise par tous les traducteurs de la $\pi \alpha ́ \alpha \delta \alpha \lambda_{1 \zeta}$ avec la panthère ${ }^{52}$. Dans la tradition physiognomonique le portrait somatique retenu pour la זó $\rho \delta \alpha \lambda ı$, et présentant un contraste général avec celui du lion, est sans doute possible celui du guépard (Acinonyx vel Cynailurus jubatus) ${ }^{53}$.

Le portrait de ces deux animaux cardinaux constitue une véritable découpe et s'attache, virtuellement, à toutes les parties visibles. La brièveté du traité aristotélicien ne permet pas de traduire chacun des attributs anatomiques du lion et du guépard dans des équations singulières, et aucun traité ultérieur ne développe systématiquement et exhaustivement l'homologie anatomique avec les animaux ${ }^{54}$. Ces indications montrent néanmoins que la physiognomonie est analytique et ne se contente pas d'allusions générales. Dans le cours de l'exposé si la référence à l'animal est synthétique pour le niveau psychique, elle est analytique et particulière pour le niveau somatique, dans la mesure où elle établit un rapport localisé entre des parties homologues de l'homme et de l'animal.

\section{2. les moriotypes}

21 Dans tous les traités l'analyse topologique et la combinatoire moriologique est essentielle ${ }^{55}$ et s'exprime en particulier dans le refus d'une assimilation globale d'une configuration humaine à une configuration animale ${ }^{56}$. La similitude étant locale et partielle il n'existe pas d'homme-lion, mais des hommes arlequins à facettes multiples, et dont le sens même n'est pas dans la somme des parties et de leur valeur, mais dans une expression synthétique qui hiérarchise les signes ${ }^{57}$. Dans la fabrique somatique l'homme se caractérise par une pluralité de similitudes ${ }^{58}$, d'où l'importance, sans doute, de la « bonne articulation" (somatique et psychique) des natures. Sur ce point le lion positif et le guépard négatif s'opposent radicalement : le premier est $\dot{\alpha} \rho \theta \rho \tilde{\omega} \delta \varepsilon \varsigma$ (Phgn. 809 b 31) tandis

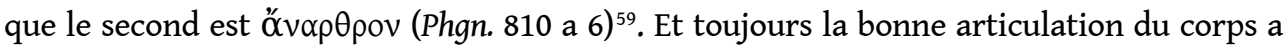
pour corollaire la vigueur et qualité psychique ${ }^{60}$, l'Anonyme latin reprenant souvent le terme grec ( $\delta i \eta \rho \theta \rho \omega \mu \varepsilon ́ v o v)$, qu'il glose par les expressions honeste dispositum et sejunctum, clarum, solidum et discretum et dont il donne comme traduction morale virile, optimum, optimi ingenii indices (AnL 51, 61, 69).

22 Adamantios, qui pratique avec retenue la méthode dite zoologique, insiste sur la précision nécessaire de ces correspondances: "il faut déterminer la nature [d'un homme] en

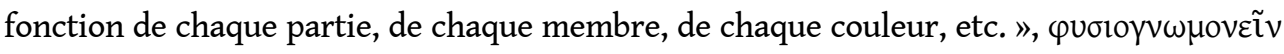

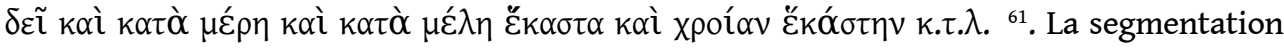
du corps animal, en unités physiques ( $\mu$ ópl $\alpha$ ) immédiatement convertibles chez l'homme se fait sans difficulté et peut compter sur deux complicités: la première est d'ordre théorique et consiste dans l'idée ancienne d'une homologie anatomique entre l'homme et l'animal, dont Aristote fait un principe de méthode générale et le fondement de l'anatomie comparée ${ }^{62}$; la seconde est d'ordre linguistique et tient à l'assimilation 
réalisée dans et par la langue des parties animales et humaines grâce à un vocabulaire descriptif unique et commun. Il y a donc une grande continuité entre l'analyse et la sémiologie physiques dans le corpus zoologique d'Aristote et celles des traités

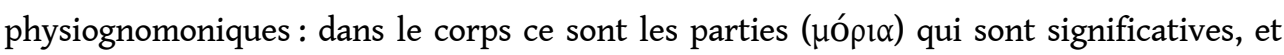
elles signifient, au-delà de leur structure immédiate, une fonction, un mode de vie, des besoins, un type de socialité, un tempérament. Dans l'homme même la taille et la forme

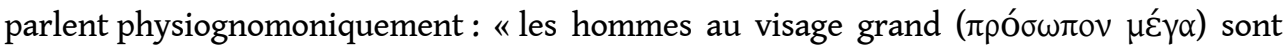
lents $(\beta \rho \alpha \delta$ v́ $\varepsilon \rho \circ 1) »^{63}$. L'absence dans nos traités de références aux parties internes, pourtant les plus révélatrices de l'intelligence et du caractère selon Aristote, est inhérente au code même de la discipline et ne marque pas non plus un écart théorique entre les deux approches ${ }^{64}$.

\section{3. la révision du schéma classique des méthodes}

Le schéma traditionnel en trois branches proposé par les auteurs anciens pour la

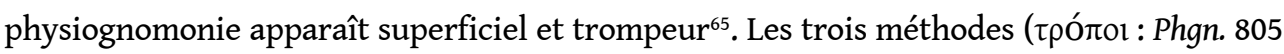

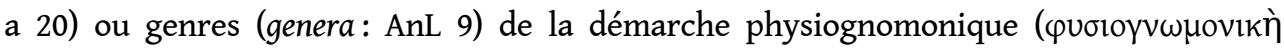

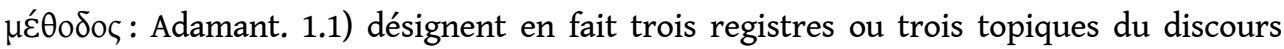
physiognomonique, mais ne constituent pas en soi trois procédés différents. Les animaux,

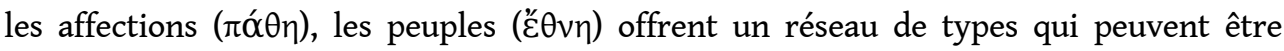
utilisés de façon métaphorique ou analogique. Mais, si l'on distingue les pratiques discursives de la physiognomonie (qu'elle soit méthodique ou clandestine) selon la fonction des modèles et la nature des équations, un clivage décisif apparaît entre une méthode (analogique) globale et une méthode analytique :

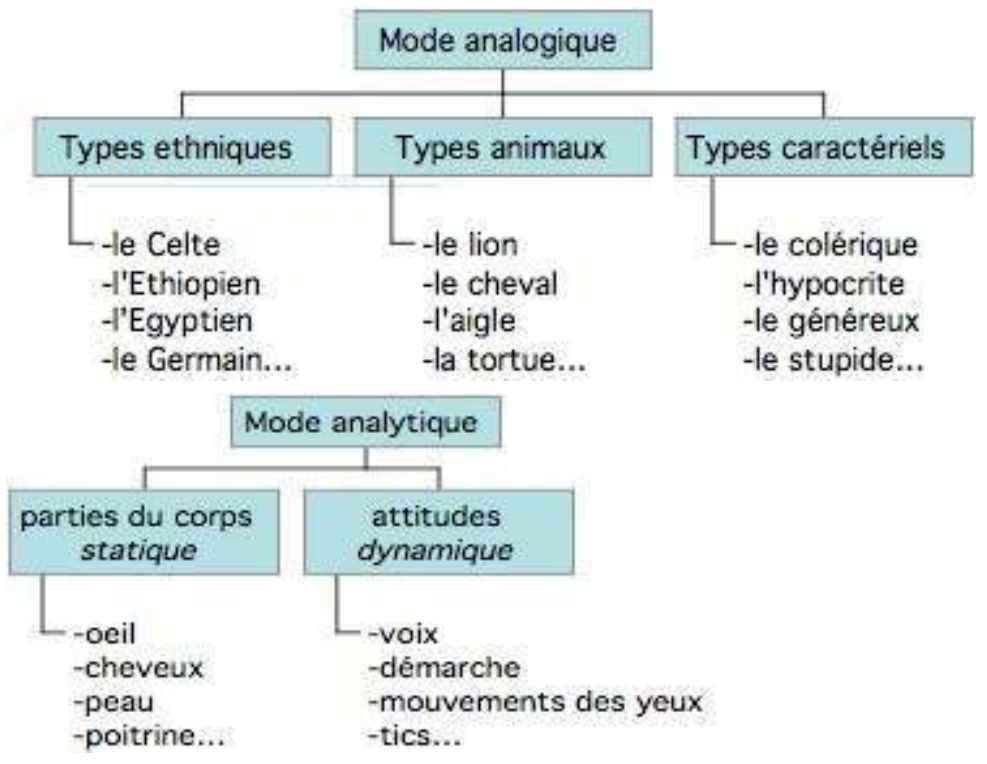

Maniée grossièrement la référence animale peut relever du mode analogique; mais dans les traités c'est généralement le mode analytique qui est développé et s'appuie sur l'aspect extrême qu'offrent les traits animaux pour identifier en l'homme son discret semblable. Ce que l'on appelle «méthode zoologique » en physiognomonie ne correspond donc pas à une modalité précise et commune de référence à l'animal et il y a une différence entre une homologie réglée et un air de famille. 


\section{4. la correspondance et la hiérarchie des parties}

Si l'on prend la peine d'étudier quelles sont les parties marquantes, significatives, dans l'analyse physiognomonique du corps on observe une focalisation de l'attention sur certaines zones privilégiées. Si tout est signe dans le corps de l'homme ${ }^{66}$ tout n'est pas signe dans le corps de l'animal ${ }^{67}$, mais la règle implicite du transfert homologique (il n'y a rien de commun entre la jambe de l'un et le front de l'autre) tend à projeter sur le corps animal la hiérarchie anatomique valable pour l'homme. Deux mesures permettent d'apprécier l'importance particulière du critère zootypique dans la lecture anatomique : le volume de texte consacré à chaque partie somatique, et les parties pour lesquelles le physiognomoniste recourt au critère zootypique de préférence aux autres critères. Le calcul pour le traité aristotélicien donne le résultat suivant : visage (20\%), yeux (11\%), mouvement des yeux (4\%), pilosité faciale (6\%), cou et tronc (18\%), membres (9\%), mouvements $(6 \%)$, teint $(8 \%)$, pilosité générale $(4 \%)$, taille $(10 \%)$, voix $(4 \%)$; et dans l'ouvrage d'Adamantios : yeux (57\%), visage (17\%) cou et tronc (11\%), membres (13\%), ventre (2\%). Ces chiffres montrent une importance exceptionnelle de la tête ( $41 \%$ et $74 \%$ ), conforme aux déclarations théoriques des auteurs qui font de la face et des yeux la zone où les signes sont les plus nombreux et flagrants: "Les signes les plus clairs

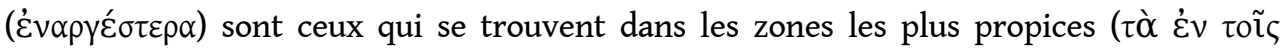

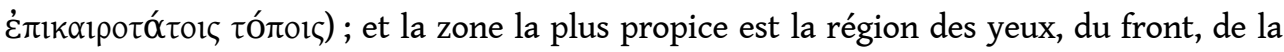
tête et du visage ; puis vient la région de la poitrine et des épaules, puis les jambes et les pieds; et en dernier la région du ventre $»^{68}$.

Cette hiérarchie des parties vaut pour l'homme comme pour l'animal. Or si l'on considère dans les Physiognomonica aristotéliciens l'usage des critères et la part relative que prend chacun d'eux dans l'élucidation des traits, en fonction du lieu du signe, il apparaît que le physiognomoniste recourt au critère de la similitude animale précisément lorsqu'il aborde les zones les plus propices : la tête et la face. C'est à partir du cou que le corps animal devient humainement le plus parlant.

Etude physiognomonique
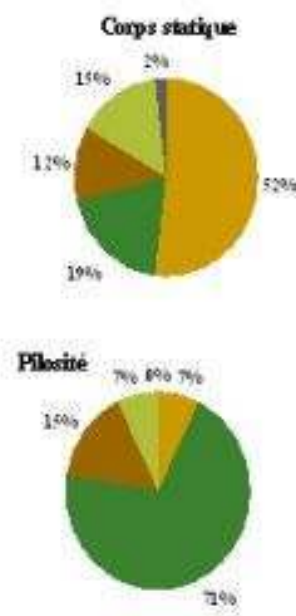
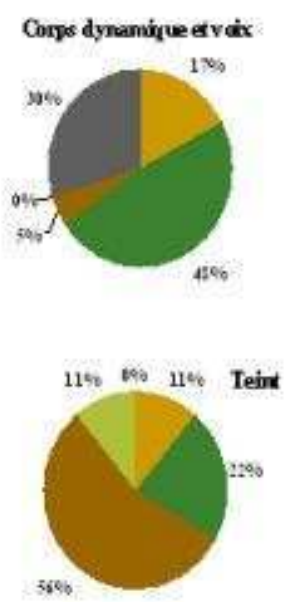

Masculin Fém

Zootypes

Pathotypes

Ethotypes

Comenance

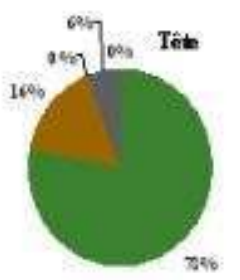




\section{5. la communauté du visage} importante car Aristote, dans ses œuvres biologiques, refuse explicitement à l'animal un visage : «La partie située sous le crâne reçoit le nom de 'visage' ( $\pi \rho$ ó $\omega \pi$ rov) uniquement

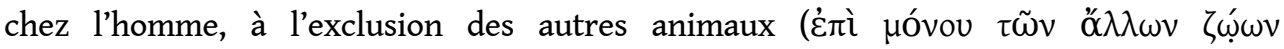

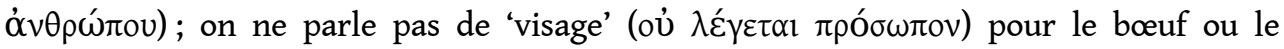
poisson $»^{69}$. L'usage d'Aristote est rigoureux et les seuls animaux pour lesquels il emploie le terme de 'visage' en dehors des Physiognomonica sont les singes (HA 502 a 20), et une fois le caméléon parce qu'il a comme un visage de babouin (HA 503 a 18:

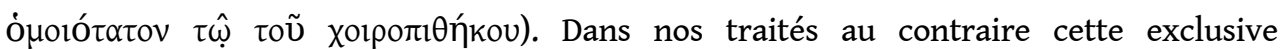
terminologique tombe et tous les animaux acquièrent, par vertu physiognomonique, un visage que la biologie leur refusait: "ceux qui ont un visage bien en chair (oi tò $\pi \rho$ ó $\sigma \omega \pi \circ v \sigma \alpha \rho \kappa \tilde{\delta} \delta \varepsilon \varsigma)$ sont paresseux ; cela renvoie aux bœufs [...] ; ceux qui ont un visage

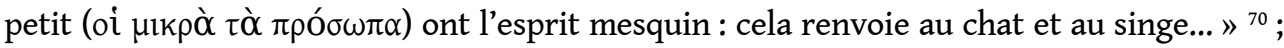
et «dans chaque espèce animale la femelle a le visage plus étroit ( $\sigma \tau \varepsilon v o \pi \rho \circ \sigma \omega \pi$ tr $\varepsilon \rho v$ ) que le mâle » (Phgn. 809 b 4).

n'est pas seulement la biologie qui refuse un visage aux bêtes, mais la philosophie également, et la morale, car l'octroi de ce privilège symbolique a des répercussions énormes $\mathrm{du}$ point de vue de l'implicite philosophique et moral. Interface psychosomatique le visage, comme le rappelle Aristote, est en effet nommé ainsi en raison de la position de la face par rapport au monde et de la verticalitét ${ }^{71}$. Autrement dit il n'est pas une partie anatomique comme les autres et ne répond pas seulement à la

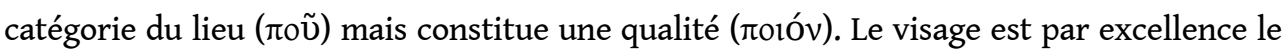
lieu de jonction du $\sigma \tilde{\omega} \mu \alpha$ et de la $\psi v x \eta ́$ et la suppression du tabou linguistique dont Aristote l'avait marqué montre que la physiognomonie exploite et radicalise l'anatomie comparée en adoptant un principe de convertibilité généralisée. L'humanisation de l'animal par le corps, qui s'accompagne d'une humanisation psychique et morale a naturellement pour effet, récursivement et réciproquement, de suggérer une animalité profonde de l'homme.

\section{Conclusion}

Aristote, dans l'antiquité, est au coeur du développement du thème de l'affinité entre homme et animal. Cette affinité est perçue à travers différents types d'interaction et de correspondances sémiologiques, et la physiognomonie contribue, de manière spéciale, à l'exprimer, en particulier à travers le rapport étroit et détaillé qu'elle établit entre les corps animal et humain. Dans les traités de physiognomonie, le modèle animal connaît deux niveaux opératoires: (1) l'idéaltype animal, qui propose à la fois une formule psychologique sommaire et un patron somatique extrême ; (2) l'archétype moriologique, qui offre un élément de lecture précis, chargé de la même signification primaire que le trait moral animal ${ }^{72}$, au nom d'une analogie poussée localement jusqu'à l'assimilation. Dans cet échange de bons procédés chacun partage ce que l'autre possède aussi, avec moins de clarté ou plus de finesse. L'homme est élucidé et l'animal moralisé, le revers discret de "l'anthropomorphisme»-qui n'est rien d'autre souvent que la vision humaine, et non angélique, du monde, acclimaté à notre mesure et raison- étant en 
l'occurrence une bestialisation de l'homme. Mais les traités ne s'interrogent pas sur les conséquences, pour la morale, de cette solidarité entre un homme, irresponsable de son corps, et un animal qui caricature brutalement l'esprit de son semblable. L'étho-logique de la physiognomonie ne peut conduire à une éthique. Elle a, bien plus, rapport à la biologie et, dans ses équations motivées, à la zoologie ${ }^{73}$.

$\mathrm{Au}$ reste, l'analogie animale n'a rien d'une illusion anthropomorphique. L'anatomie comparée est scientifiquement fondée, et, dans le domaine psychologique -puisque, encore une fois, la physiognomonie ne s'intéresse pas à la morale mais au psychisme- les comportements animaux sont, en certains cas, comme ceux des hommes, déterminés par des processus mentaux réguliers et propres : le lion est effectivement fort et courageux et le lièvre lâche et craintif (Vogt 1999, 161). Dans quelle mesure le courage du lion est-il «métaphorique»? Là encore, le partage, à l'intérieur d'Aristote, se fait entre la perspective éthique, d'une part, et la perspective zoologique et physiognomonique, de l'autre ${ }^{74}$. Au fond, l'audace -ou le scandale- de la physiognomonie ne réside pas tant dans ce qu'on nomme vaguement la "méthode zoologique (la continuité animale et l'unité du (oov) que dans son principe même qui pose l'unité psycho-somatique de l'homme et cherche la cohérence globale de l'ethos. Mais n'est-ce pas, sourdement, la langue ellemême qui nous physiognomonise ou nous rend physiognomonistes, en mettant en commun un vocabulaire unique pour décrire le corps et l'esprit, ne connaissant aucune frontière linguistique entre les manifestations psychiques et les expressions somatiques? L'ethos, pivot conceptuel de cette unité du signe psycho-somatique est-il le dehors de l'esprit ou le dedans du corps? Semblablement de l'homme et de l'animal le corps se peut-il dire ou percevoir autrement que comme une nature unique?

\section{BIBLIOGRAPHIE}

\section{Principales éditions et traductions récentes du corpus physiognomonique}

ANDRÉ, J. (ed. \& trad. fr.) 1981. Anonyme latin. Traité de Physiognomonie, Belles Lettres, CUF.

DEGKWITZ, A. (trad. all. et comm.) 1988. Die pseudoaristotelischen 'Physiognomonica', Traktat A. Übersetzung und Kommentar, Heidelberg.

FERSTER, R. (ed.) 1893. Scriptores Physiognomonici Graeci et Latini, Leipzig, 2 vol.

HETT, W.S. (ed. \& trad. angl.) 2000 [1936]. Physiognomics in Aristotle. Mino works, Harvard, Loeb.

MANZANO, T. Martinez (trad. esp.) 1999. Pseudo Aristóteles. Fisiognomía, Gredos 270.

PACK, R.A. (ed.) 1974. « Auctoris incerti De physiognomonia libellus », AHMA (Archives d'histoire doctrinale et littéraire du Moyen-Age) XLIX, p. 113-138.

RAINA, G. (trad. ital.) 1993. Pseudo Aristotele, Fisiognomica. Anonimo latino, Il trattato di fisiognomica, Milan. 
VOGT, S. (ed., trad. et comm. all.) 1999. Aristoteles. Physiognomonica. Aristoteles. Werke in deutscher Übersetzung. Band 18, Opuscula, t. 6, Berlin.

\section{Études critiques}

AGRIMI, J. 1993. « Fisiognomonica e Scolastica », Micrologus. Natura, Scienze et Società medievali, I, p. 235-271.

AGRIMI, J. 1996. «Physiognomica : nature allo specchio ovvero luce e ombre », Micrologus. Natura, Scienze et Società medievali, IV, p. 129-178.

ASMUS, R. 1906. « Vergessene Physiognomika »,Philologus 65, p. 410-424.

BALTRUSAITIS, J. 1995 [1983]. «Physiognomonie », in :Aberrations, Les perspectives dépravées I, Flammarion, p. 13-85.

BONITZ, H. 1867. « Über пó $\theta$ oৎ und пó $\theta \eta \mu \alpha$ im Aristotelischen Sprachgebrauch. Aristotelische Studien V ", Sitzungberichte der Östereischicher Akademie der Wissenschaften in Wien, historische Klasse , 55, p. 51 sq. (= Aristotelische Studien, Hildesheim 1969, p. 325 sq.)

BONITZ, H. 1870. Index aristotelicus, Berlin.

CARO BAROJA, J. 1988. Historia de la Fisiognómica. El rostro y el carácter, Madrid.

DAGRON, G. 1987. « Image de bête ou image de dieu. La physiognomonie animale dans la tradition grecque et ses avatars byzantins ", in : Poikilia, Études offertes à Jean-Pierre Vernant (Détienne M., Loraux N., Mossé C. \& Vidal-Naquet P. ed.), p. 69-80.

DIERAUER, U. 1977. Tier und Mensch im Denken der Antike, Amsterdam.

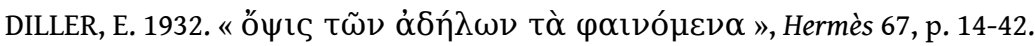

DUMONT, M. 1984. «Le succès mondain d'une fausse science, la physiognomonie », Actes de la recherche en sciences sociales 54 , p. 2-30.

EVANS, E.C. 1969. Physiognomics in the ancient World. American Philosophical Society, new series, $59(5)$.

FCERSTER, R. 1926. «Zu den griechischen Physiognomikern », Philologus 81, p. 236 sq.

FCRSTER, R. 1888a. « De aristotelis quae feruntur physiognomonicorum indole ac condicione »,in : Philosophische Abhandlungen, Martin Hertz zum siebzigsten Geburtstag, Berlin.

F@RSTER R. 1888b. « De Loxi Physiognomonia », Rheinisches Museum 43, p. 505-511.

FCERSTER, R. 1884. Die Physiognomik der Griechen, Kiel.

FCERSTER, R. 1881-1882. « Dissertatio de Aristotelis quae feruntur physiognomicis recensendis ", Schriften der Universität Kiel 28.

KIILERICH, B. 1988. «Physiognomics and the iconography of Alexander ", Symbolae Osloenses LXIII, p. 51-66.

LAPINI, W. 1992. « Il nome, la maschera e l'idiota », Sandalion 15, p. 53-101.

LAURAND, V. 2005. « Les hésitations méthodologiques du Pseudo-Aristote et de l'Anonyme latin », in : La physiognomonie. Problèmes philosophiques d'une pseudo-science (C. Bouton et V. Laurand, eds), Kimé, p. 17-44.

LAURAND, V. 2006. «Du morcellement à la totalité du corps signe : l'alphabet et la lecture dans les traités antiques de physiognomonie ", Actes du colloque international Celtic Conference in 
classics, panel « Penser et représenter le corps », Presses Universitaires de Rennes, sous la direction de F. Prost et J. Wilgaux (à paraître).

LLOYD, G.E.R. 1983. « The development of zoological taxonomy », in : Science, folklore and ideology. Studies in the life sciences in ancient Greece, Cambridge University Press, p. 7-57.

LOMBARDI, M. 1999. « Paradigmi caratteriologici ed osservazione empririca nei Caratteri di teofrasto e nei Physiognomonica Pseudoaristotelici », Rivista di Cultura Classica e Medioevale 1.

MacC. ARMSTRONG, A. 1958. « The methods of the Greek Physiognomists ", Greece and Rome, 5 (1),p. 52-56

MANETTI, G. 1987. Le teorie del segno nell'antichità classica, Milan.

MARGANNE, M.-H. 1988. « De la physiognomonie dans l'Antiquité gréco-romaine », in Ph. Dubois \& Y. Winkin (ed.)Rhétoriques du corps, Bruxelles, De Boeck-Wesmael, p 13-24.

MEGOW, R. 1963. « Antike Physiognomielehre », Das Altertum 9, p. 2218-2223.

PACK, R.A. 1941. "Artemidorus and the Physiognomists ", Transactions and Poceedings of the American Philological Association 72, p. 321-334.

ROCCA-SERRA, G. 1997. " Homme et animal dans la physiognomonie antique », in : L'animal dans l'Antiquité (B. Cassin \& J. L. Labarièrre, eds) Paris, Vrin, p. 133-139.

RODLER, L. 2000. Il corpo specchio dell'anima. Teoria e storia della fisiognomica, Milan.

SASSI, M.M. 1993. « Physiognomonica », in Lo spazio letterario della Grecia antica I, 2 (Cambiano G., Canfora L., Lanza D. ed.), p. 431-448.

SASSI, M.M. 1988. La scienza dell'uomo nella Grecia antica, Turin.

SCHMITT, J. 1941. «Physiognomik », RE XX, 1, p.1064-1074.

TSOUNA V. 1998. « Doubts about other minds and the science of Physiognomics », Classical Quarterly 48 (1), p. 175-186.

WOTKE F. 1949. « Panther » in RE XVIII 2b 747-767.

ZUCKER, A. 2006. « La sémiologie animale dans les traités de physiognomonie antique », in : Akten der internationaler altertumswissenschaftlicher Tagung 'Mensch und Tier in der Antike : Grenzziehung und Grenzüberschreitung', Rostock 6-10 März 2005 (sous presse).

\section{ANNEXES}

\section{ANNEXE 1 : Les animauX dans les traités de Physiognomonie}

ARISTOTE, Physiognomonica $=1$ (méthode et généralités), 2 (les parties du corps), 3 (les caractères) ; 4 (méthodes et généralités), 5 (les critères), 6 (les parties du corps).

POLÉMONarabe, De physiognomia liber =1-52 (les parties du corps), 53-70 (les caractères ). [sauf chap 2 les caractères des animaux ; chap 31-35 les ethnotypes ; chap. 68-70 quelques études de cas]. 
ADAMANTIOS Physiognomonica = 1.1-4 (méthode et généralités), 1.5-23 (les parties du corps : les yeux), 2.1-3 (généralités), 2.4-42 (les parties du corps autres que les yeux), 2.43-60 (les caractères), 2.61 (conclusion) [sauf 2.31-32 (les ethnotypes) à propos du teint].

ANONYME LATIN, De physiognomia liber = 1-12 (généralités), 13-80 (les parties du corps), 81-88 (compléments de Loxos et Aristote), 89-115 (les caractères), 116-117 (remarques sur le milieu), 118-131 (les zootypes), 132-133 (conclusion).

\section{ANNEXE 2 : Les parties des animaux}

\section{Références à des moriotypes animaux (tête/corps/dynamique) dans Arist. Physiogn. [caractères droits] et les traités postérieurs [italiques ].}

Bouche $(\sigma \tau o ́ \mu \alpha):$ très ouverte = monstres marins ( $(n ́ \tau \eta):$ Adamant. 2. 24, p. 374 \& Ps.Pol. Cheveux $\left(\tau \rho \imath \omega^{\prime} \mu \alpha \tau \alpha\right)$ : durs = lion, sanglier, oiseaux (806 b 6-18); souples = cerf, lièvre, mouton, oiseaux (ibid.); bouclés à l'extrémité = lion ( 812 b 33); ch. du front recourbés vers l'arrière = lion (812 b 34) ; blonds = lion (812 a 15) ; roux = renard (812 a 16) ; [poils] poilus sur le ventre = oiseaux ( $806 \mathrm{~b} 18)$; poilus sur les mollets = boucs ( $812 \mathrm{~b} 13$ \& Pol. 43, p. 252) ; poilus sur la poitrine et le ventre = oiseaux (812 b 14); poilus sur les épaules = oiseaux : 812 b 19 (Pol. 45, p. 253 ; cf. AnL 73 avium mores) ; poilus sur le dos = bêtes sauvages $(\theta \eta \rho i ́ \alpha): 812$ b 21 ; poilus sur la nuque = lion : 812 b 23 (Pol. 46, p. 254 ; cf AnL $73)$; ch. raides et noirs ou ternes et d'un rouge délavé = cochon (AnL 14); ch. drus, avancés sur le front = ours AnL 14 ; ch. descendant à mi-front et ramenés de chaque côté = cheval AnL 73 ; ch. souples $($ coma mollis $)=$ certains oiseaux et quadrupèdes, Pol. 40, p. 250); ch. rouges = bêtes sauvages, ferae, Pol.41, p. 250. NB : Poils = cheveux (poils longs et abondants : bestiae, Pol. 42, p. 252 ; pour les oiseaux, cheveux = plumes). Cou : épais = taureau (811a 13); grand sans être trop épais $=$ lion (813 a 14); long et fin $=\operatorname{cerf}(811$ a 16) ; raccourci $=$ loup $(811$ a 17$) ;$ large $=$ porc (AnL 53) ; Solide, large, rigide = loup, singe (AnL 55). Front : petit = cochon, 811 b 28 (AnL 17) ; trop grand = bœuf ( 811 b 29) ; rond = âne ( 811 b 30) ; plutôt long et aplati = chien ( 811 b 31 ; allongé -oblonga- AnL 17) ; rectangulaire = lion (811 b 33) ; ténébreux (froncé,

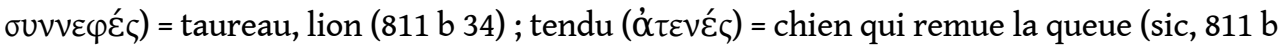
35 ; cf. AnL 17 : peau du front lâche, dilaté et comme souriante = chiens caressants -blandientes canes-) ; cf. resserré vers le crâne = lion (AnL 83). Lèvres :fines et souples ( $\chi \alpha \lambda \alpha \rho \alpha ́)$ aux jointures avec un surplis aux jointures qui abaisse la lèvre supérieure sur l'inférieure = lion (cf. Pol. 25, p. 224 ; AnL 48) et chiens puissants (811 a 19) ; minces, sèches, proéminentes au niveau des canines = cochons (811 a 22 ; Adamant. 2. 24, p. 374 ; Ps.Pol. ; cf. AnL 48 ; cf. Pol. 25, p. 226 : lèvres et mâchoires épaisses) ;épaisses, la supérieure avançant sur l'inférieure = âne et singe (811 a 24) ; l. supérieure et gencive en avant =chien (811 a 26 ; cf. AnL 48 ; Adamant. 2. 24, p. 374 ; Ps.Pol.) ; lèvres qui pendent mollement = vieux ânes, vieux chevaux (AnL 48); lèvres épaisses et large bouche = crocodile, Pol. 25, p. 226. Menton : pointu (ou pointe du menton poilue) $=$ chien (812 b 24); petit = serpent (AnL 52). Nez : camus $=\operatorname{cerf}(811$ b 2) ; bout du nez épais = bœuf ( 811 a 28$)$; pointe du nez épaisse $:$ cochon (811 a 29 ; cf. AnL 51) ; pointe du nez effilée : chien (811 a 31) ; pointe du nez ronde et obtuse = lion (811 a 32 ; AnL 51 lion et chien de race); nez bien décalé au niveau du front = lion (AnL 51) ; camus dès la naissance = lion (AnL 83) ; épais, long, obtus, fort = chien et lion (Pol. 26 
p. 228); mou, obtus, rong, fort = lion et chien de race (Adamant. 2. 25, p. 375 ; Ps.Pol.) ; pointe du nez fine = oiseau (811 a 33); nez légèrement crochu à partir du front et ensuite droit = corbeau (811 a 34); cf. nez fin à la naissance = corbeau (AnL 83) ; nez crochu décalé $(\delta ı \eta \theta \rho \omega \mu \varepsilon ́ v \eta v)$ par rapport au front = aigles (811 a 36) ; nez incurvé (vers l'intérieur) [concave, bombé] arrondi au niveau du front et dont la partie ronde se dresse vers le haut = coqs, (811 a 37); nez épais = oiseaux immondes (sordidae, AnL 51); nez long et fin = oiseaux (Pol. 26, p. 228 ; Adamant. 2. 25, p. 375 ; Ps.Pol.). Oreilles : petites = les gens sont $\pi \imath \theta \eta \kappa \omega ́ \delta \varepsilon ı$, ,

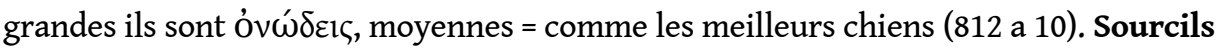
:tombant du côté du nez et montant du côté des tempes = cochon ( 812 b 26 ; Pol. 48, p. 254 ; AnL 18) ; s. abaissés tandis que les pupilles sont orientées vers le haut : chien (AnL 83). Tête : grande $=$ chien $(815$ a 5$) ;$ petite $=$ âne, $(812$ a 7$) ;$ pointue $=$ rapaces $(812$ a 8$) ; c f$. petite presque ronde = rapaces (aves, AnL 16). Grosse tête, front large, visage proéminent = bœuf (AnL16). Visage ou face : charnu = bœuf $(811$ b 4); charnu = âne et cerf $(811$ b 6) ; petit = chat et singe ( 811 b 8) ; grand = âne et bœuf ( 811 b 9). Yeux : petits = singes ( 811 b 18) ; gros = bœuf (811 b 20 ; cf. maesti : Pol. 2, p. 192) ; renfoncés = singes (811 b 22 ; Pol. 2, p. 192 ; cf. Adamant. 2. 2. p. 350); exorbités = ânes ( 811 b 23 ; Pol. 2, p. 192 ; cf. Adamant. 2. 2, p. 350 : $\pi \rho \circ \pi \alpha \lambda \varepsilon \tilde{\mathrm{l}} \varsigma$ ) ; $c f$. saillants (prompti, AnL 83) ; légèrement enfoncés = lion (811 b 26 ; Pol. 2, p. 192 ; cf. Adamant. 2. 2, p. 350) ; un peu plus renfoncés : bœuf (811 b 28 ; cf. Adamant. 2. 2,

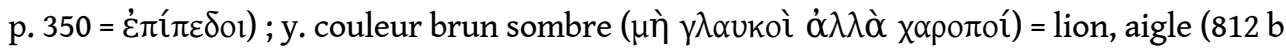
5) ; caprins = chèvres (812 b 6 ; AnL 83 ; cf. Adamant. 2. 36, p. 391 : $\mu \alpha \rho \mu \alpha \rho u ́ \sigma \sigma o v \tau \alpha-$ brillant- $)$; brillants = coqs et corbeaux ( 812 b 11 ; cf. AnL 83, ronds et brillants = coqs $)$; couleur du feu = chien $(812$ b 7 ; Adamant. 2. 36, p. $391=\pi v \rho \omega \delta \eta)$; brillants = coqs et corbeaux (812 b 11 ; cf. AnL 83 : ronds et brillants = coqs ; cf. Adamant. 2. 36, p. 391 : étincelants $\sigma \tau i ́ \lambda \beta o v \tau \varepsilon \varsigma=$ oiseaux $) ;$ mobiles = faucons $(813$ a 19) ; grands et mobiles = loups et sangliers (Adamant. 1. 11, Ps.Pol. ; pupilles petites = serpents, ichneumons, singes, renards (Adamant. 1.5 ; Ps.Pol., AnL 21) ; yeux à larges pupilles = bœuf, mouton (Adamant.1.5) ; yeux gris bleu $=$ animaux sauvages (Pol. 39, p. 246) ; y. couleur d'antimoine $=$ animaux domestiques (Pol. 39, $p$. 246).

Bassin : solide, dur avec des os biens distincts = lion (AnL 68); maigre avec une peau ridé et fine= singe (AnL 68). Corps courbé et comme cassé = chien (AnL 76); recourbé sur soi même = chien quand il flatte (Adamant. 2. 41, p. 401). Cuisses velues = boucs (AnL 87). Doigts courts et épais =

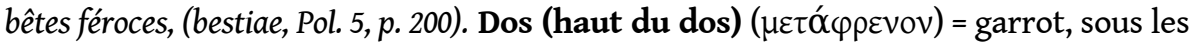
épaules (bombé, Ǔ $\pi \tau 10 \varsigma)$, convexe = cheval ( 810 b 31). Fesses $(\pi \tilde{v} \gamma \alpha)$ : peu charnues = singes ( 810 b 2 ; cf. AnL 87 : singes et ours). Flancs ( $\pi \lambda \varepsilon v \rho \alpha ́)$ : volumineux, comme gonflés d'air $=$ [bœufs et $]$ grenouilles ( 810 b 14 ; cf. AnL 65 : grenouilles). Mollets ( $\kappa \nu \tilde{\eta} \mu \alpha)$ : fins = oiseaux (810 a 32). Orteils ( $\pi \circ \delta \tilde{\omega} \nu \delta \alpha ́ \alpha \tau u \lambda \circ)$ : recourbés, et ongles aussi = rapaces $(810 \mathrm{a}$ $20)$; soudés ou palmés $=$ palmipèdes $(810$ a 22). Ongles recourbés et crochus $=$ oiseau et animaux féroces (ferae, AnL 60) ; étroits, longs, rigides = bêtes féroces (bestiae, Pol. 3, p. 198). Pieds courts et épais = bêtes féroces (bestiae, Pol. 5, p. 20).Reins peu charnus = renard (Pol. 9, p. 206).

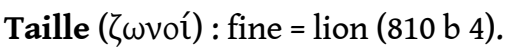

Démarche : se balançant avec les épaules tendues et dans le sens vertical = cheval (813 a 11 ; Adamant. 2. 40, p. 400 ; cf. AnL 76) ; roulant en alternance les épaules vers l'avant = lion (813 a 11 ; Adamant. 2. 40, p. 400 ; cf. AnL 76).Voix : forte et grave = âne (813 a 31) ; grave et devenant aiguë sur la fin = bœuf (813 a 32); forte et grave sans être frappante ( $\mu$ ฑ̀

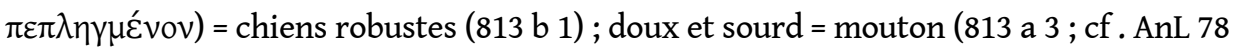

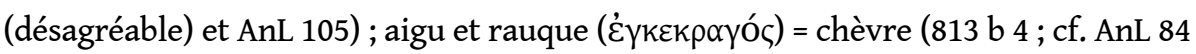


(tendue et criarde -intensa, proxime captrino balatu-) ; voir AnL 78 : voix des oiseaux; ferme, un peu assourdie, parfois grave = lions (AnL 5); cf. Pol. 52, p. 268, oiseaux, singe, chien, âne, bêtes sauvages -bestiae- ; cf. Adamant. 2. 42 : voix de chien, singe, âne, cheval, ours, mouton et autres.

\section{ANNEXE 3 : Les traits déterminants des espèces $(A n L)$}

\section{Récapitulatif des lieux du corps où se traduit chez l'homme la ressemblance avec un des quatorze animaux typiques de $A n L$ (§118-131).}

Âne > jambes, tête, oreilles, lèvres, voix ; boeuf > tête, front, yeux, bouche, naseaux, flancs, panse ; cerf > taille, membres, joues, démarche; cheval > cheveux, joues, cou, narines, lèvre inférieure ; chien > visage, bouche, corps, nez, yeux ; chouette > nez, joue, yeux, épaules, dos (strictement féminin] ; coq > yeux, tête, cou, épaules ; lion > tête, yeux, bouche, narines, cou, épaules, poitrine, hanche, cuisses, bouts des pieds et des mains, teint, cheveux ; loup > nez, sourcils, yeux, tête, corps (pilosité), cheveux, jambes ; oie > pieds, jambes, talons, nez, tête, yeux, dos ; paon > corps, tête, yeux, cou, démarche ; serpent > tête, yeux, cou, bouche, corps, poitrine, mouvement (de tête) ; singe > yeux, barbe, cou, visage ; tortue > cou, dos, pieds, visage, joues [strictement féminin]. NB : le cas du bouf est exceptionnel puisque la description physique directe est celle de l'animal et non du corps de l'homme répondant au type du bœuf.

\section{NOTES}

1. Rodler $(2000: 7)$ donne à la physiognomonie une étymologie farfelue, doublée d'une glose fautive : " come suggerisce l'etimologia, la fisiognomica è physis-onoma, conoscenza della natura, indagine circa l'intelligibilità di un reale polimorfo. Ma essa è anche un sistema rigoroso ed esatto di corrispondenze tra soma e psiche, vale a dire physi-snomos (sic), legge della natura ».

2. Sur les principales publications postérieures à l'édition de Foerster voir la synthèse ( Forschungsüberblick) et la riche bibliographie de S. Vogt (1999 : 242-281).

3. La pagination est partout celle de l'édition Foerster. Mais le texte d'Adamantios est accompagné de deux épitomés parallèles qui, dans son cas, faussent le chiffre qui doit être au moins divisé par deux.

4. Le texte a subi des altérations dont témoigne, par exemple, la mention dans le texte donné par Foerster d'une indication datable du $\mathrm{II}^{\mathrm{e}}$ siècle ap. J.C., postérieure en tout cas à Polémon (Phgn.

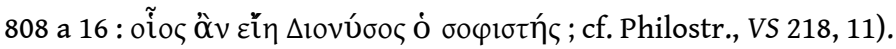

5. Il faut indiquer, bien qu'il ne s'agisse pas d'un traité de physiognomonie proprement dit, le texte de Galien Quod animi mores corporis temperamenta sequantur (Kühn, 4, 767-822) qui commente les théories philosophiques (en particulier de Platon) sur l'âme, souligne la corrélation entre les différences psychiques et somatiques, en insistant sur la théorie des humeurs (la kpõ $\sigma ı \varsigma$ étant à la

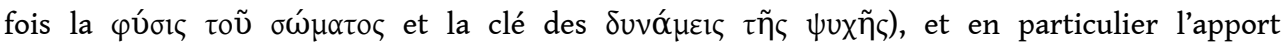
d'Hippocrate et des œuvres biologiques d'Aristote (791-795) à cette théorie, en donnant une version presque univoque du déterminisme physique (du corps sur l'esprit) et retenant (comme le médecin Loxos : Foerster 1888b) l'influence déterminante du sang dans cette action.

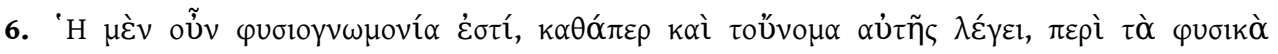

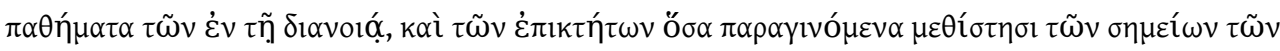

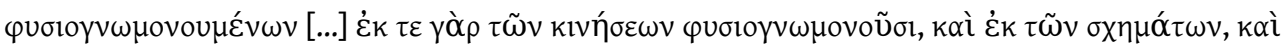




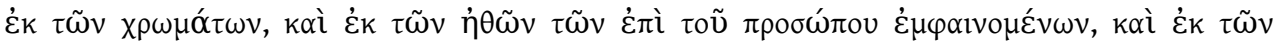

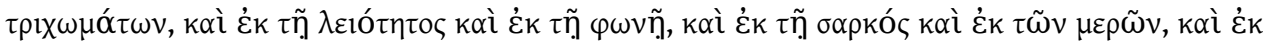

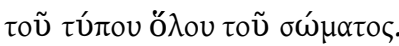

7. Le traité de Galien qui traite de l'unité psychosomatique de l'homme commence

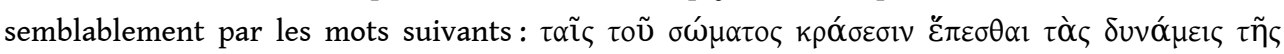
$\psi v x \tilde{n}$ s... (Galien, Quod animi mores 4, 767,1)

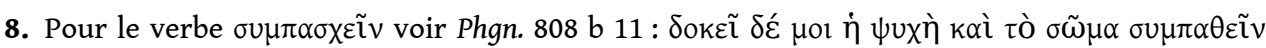

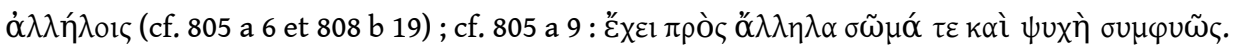

9. L'usage de ce terme dans cette connexion se trouve seulement dans la première partie du traité aristotélicien des Physiognomica (Galien employant le mot dans Quod animi mores, mais hors contexte). Les deux désignations de la composante mentale sont équivalentes et Aristote en use indifféremment, et presque en alternance : $\psi v x \eta ́$ ( $\$ 1=805$ a 6, 10, 13, 23, b 13 etc. ; $\$ 4=811$ b 11)

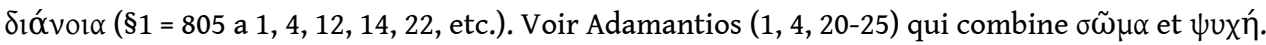

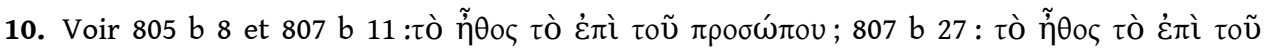

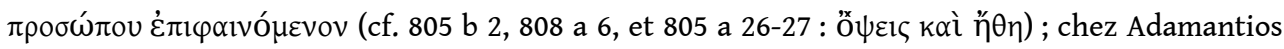

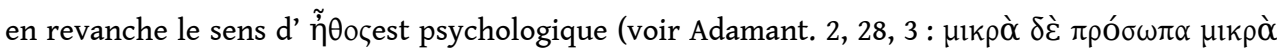

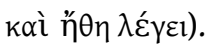

11. Anax B 21 ; voir Diller 1932.

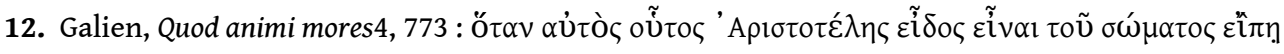

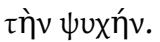

13. Cf. Auctoris incerti de physiognomonia libellus (Pack 1975 : 126) : « elegans est nature cognicio que per exteriores formas interiores investigat qualitates, id autem efficit phisiognomice compendium sciencie, dicitur autem phisiognomia a 'phisis' quod est natura, et 'gnomos' quod est divinacio ".

14. Les traités tardifs ou modernes privilégient tantôt la valeur pratique (voir AnL. 2: "profitetur itaque ex qualitate corporis qualitatem se animi considerare atque perspicere »), tantôt la portée philosophique de la discipline (voir Lavater, La physiognomonie ou l'art de connaitre les hommes d'après les traits de leur physionomie, Lausanne, 1979, p. 6 : «La physiognomonie est la science, la connaissance du rapport qui lie l'intérieur et l'extérieur, la surface visible à ce qu'elle couvre d'invisible [... elle] se propose de connaître les signes sensibles de nos forces et de nos dispositions naturelles »).

15. C'est là un des aspects de la nature tautologique du discours physiognomonique (voir infra et Zucker 2006).

16. Voir aussi la formulation précieuse qui en est donnée dans le dernier chapitre des Premiers analytiques, dont il sera de nouveau question plus loin : « les affections naturelles provoquent un

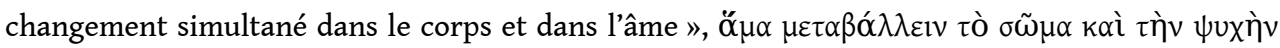

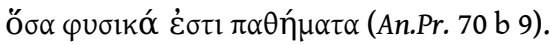

17. A tout le moins s'agissant de traits constants de part et d'autre (Phgn. 806 a 8).

18. Ce syllogisme est explicite chez Della Porta (De humana Physiognomia, Naples, 1586; voir Baltrusaitis 1995 : 23).

19. Aristote propose pour ce faire une indispensable base théorique, tant pour l'anatomie (PA)

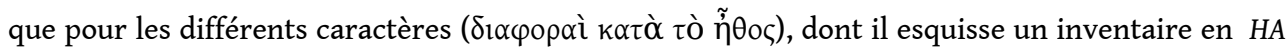
$488 \mathrm{~b} 12$ sq.

20. Lavater, respectueux de cette logique, propose dans son traité de physiognomonie ( Physiognomonische Fragmente, Leipzig et Winterthur, 1775-1778) de nombreuses planches d'étude de physiognomonie d'animaux (Baltrusaitis $1995: 61$ sq.).

21. C'est le sens du long chapitre consacré par Polémon au portrait moral de 93 animaux (Forster 1893, I 170-198), dont la liste se conclut par l'avertissement suivant : scias autem hac animalium 
eorumque morum commemoratione id intendi, ut physiognomoni exemplaria sint e quibus de eorum moribus iudicet qui iis forma similes sunt.

22. Phgn. 805 a 15-17.

23. Les études sur ce point partent d'un passage des Premiers Analytiques 70 b 7-37 dans lequel, à l'occasion d'une réflexion sur le signe et à propos de la définition de l'enthymème, Aristote énonce les trois réquisits d'une science physiognomonique : il faut que $\psi v x \eta ́$ et $\sigma \tilde{\omega} \mu \alpha$ soient en

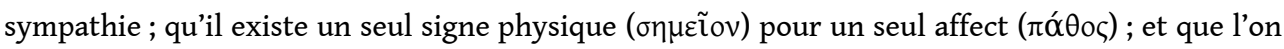
puisse déterminer pour chaque espèce animale le pathos (psychique) et son symptôme

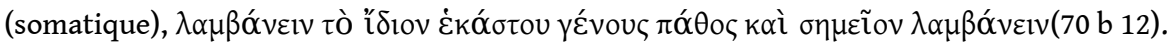

24. Le premier chiffre des parenthèses correspond au nombre d'animaux pour lesquels l'auteur donne une détermination somatique ou psychique significative, tandis que le second recense tous les animaux cités (dont le nom figure en italiques dans les listes) dans le texte.

25. Ce chiffre doit être porté à 93 si l'on tient compte du dédoublement de certains genres en espèces, mais le document de travail étant une traduction latine ancienne d'un original arabe, il faut rester prudent sur ces chiffres, comme sur les identifications zoologiques.

26. Pol. 23r (Foerster 1893, I, 182) : delphinus ludicrus concupiscens innocuus. Carcharias malignus protervus perfidus fugax audax.

27. AnL 118-131; voir Annexe 3.

28. Le corpus d'animaux de référence peut être encore plus chétif, comme l'atteste Aristote: "Ainsi, souvent, par moquerie, on compare certaines personnes qui ne sont pas parmi les belles, soit à une chèvre soufflant du feu, soit à un mouton cossant. Et certain physionomiste ramenait tous les traits à ceux de deux ou trois animaux, et emportait souvent l'adhésion de son

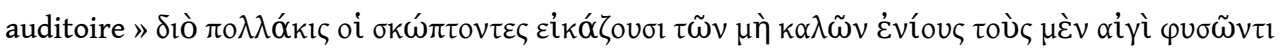

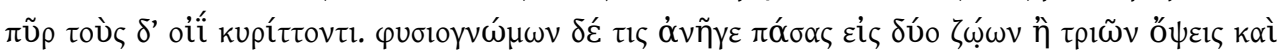

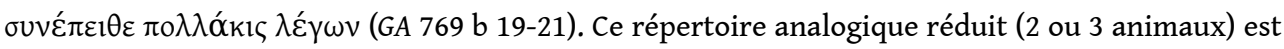
bien conforme à un motif lyrique, celui de la caractérologie par analogie animale, représenté par Sémonide d'Amorgos (VII) ou par Phocylide (VI) dans les fameux iambes (respectivement 7 et 2 [Diehl]) où l'on a 7 ou 4 modèles animaux. L'indication d'Aristote renvoie à un usage ludique, dérisoire (il vise les personnes laides) de la comparaison, et assimile le physiognomôn à un caricaturiste. Sous cette forme archaïque non formalisée la physiognomonie est l'art persuasif mais sans autre prétention que physique, à ramener un visage ou une morphologie (eidos) à un type animal.

29. Voir à ce titre les remarques pertinentes de Galien (Quod animi mores 4, 771) sur la dénomination $\kappa \alpha \tau^{\prime} \dot{\varepsilon} \xi o x \eta ́ v$ (par excellence), ou désignation d'une espèce par le nom du genre entier.

30. AnL 8 ; voir AnL 46 où les noms soulignés sont repris.

31. Le sens de ce mot apparaît très difficile à cerner et, comme on l'a dit plus haut, l'acception générale est psychique, tandis que la valeur somatique paraît restreinte aux Physiognomica (cf. Bonitz $1870: 316$ b).

32. HA 488 a 12-24 (trad. P. Louis). Le simplisme du portrait psychique contraste avec la finesse éthologique déployée ailleurs par Aristote qui note, chez une même espèce, des dispositions affectives et morales très variables, y compris dans les déterminations de base comme le courage (voir infra): « les éléphants diffèrent egalement entre eux, relativement au courage, de manière

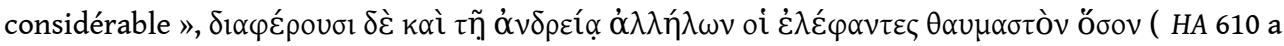
18).

33. Sur le lion, la complexité de son caractère et la distinction de deux types physiques voir e.g. HA 629 b 8-630 a 2; sur les espèces chevalines -que la littérature hippiatrique, depuis Simon d'Athènes $\left(\mathrm{V}^{\mathrm{e}}\right)$ et Xénophon ( $\left.\mathrm{IV}^{\mathrm{e}}\right)$ détaille suffisamment- signalons l'élégante esquive de Polémon qui, après avoir tiré le portrait du cheval robustus, patiens, fortis, etc. poursuit : secundum specierum diversitatem eius status differunt, sed proprietates eius prolixius describere huius loci non est (Pol. 23r in 
Foerster 1893 I, 182) ; pour le chien (au nez long et fin, aux sourcils bas, à la tête longue selon les Physiognomonica ; voir Annexe 2) qu'on songe à l'allure peu commune de trois races célèbres dans l'antiquité : le bichon maltais, le lévrier italien (de Laconie) et le dogue du Tibet. Seul l'Anonyme latin semble distinguer le cas du bichon (chien de Méléda) si c'est bien le sens qu'il faut donner à blandientes canes (AnL 17).

34. Si la méthode zoologique se fondait sur le savoir éthologique elle ne pourrait pas offrir de modèle puisque les variétés animales sont distinguées somatiquement et psychiquement. Ainsi parmi les trois espèces de hérons l'une est cendrée et industrieuse ( $\varepsilon \dot{u} \mu \eta ́ \chi \alpha \vee \circ \zeta)$, tandis qu'une

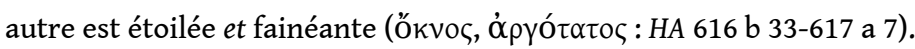

35. Arist., Phgn. 805 a 11. «Jamais, fait écho Della Porta dans la Fisionomia del uomo (29), la nature ne fait un animal qui a le corps (corpo) d'un animal et l'esprit (animo) d'un autre : c'est-à-dire un loup, ou un agneau, qui a l'âme (anima) d'un chien ou d'un lion ; mais le loup et l'agneau ont l'âme d'un loup et d'un agneau. D'où il s'ensuit nécessairement qu'à un certain corps convient une certaine âme, correspondant à son espèce ".

36. Adamantios (2. 2) exprime nettement -avec d'autres mots- cette relation de traduction

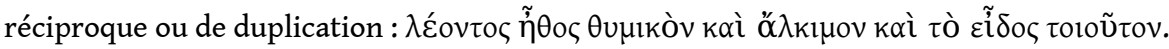

37. Cf. Rodler 2000 : 39, qui considère, elle, la « simplicità della struttura animale » comme un fait.

38. Voir les développements du livre IX de HA, en particulier HA 610 b 20-22 (et 608 a 13 et a 31).

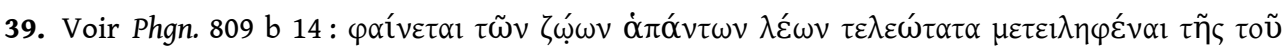

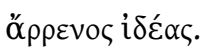

40. Phgn. 810 a 8-11; et plus haut (809 b 36 : «parmi les animaux d'allure courageuse (ou virile)

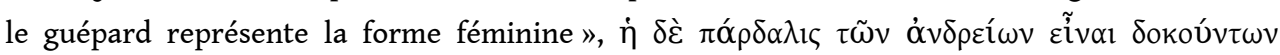

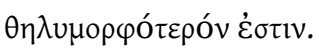

41. HA 608 a 33 : «Les femelles ont dans toutes les espèces moins de bravoure que les mâles, sauf chez le guépard et l'ours: dans ces espèces c'est la femelle qui semble être la plus virile ",

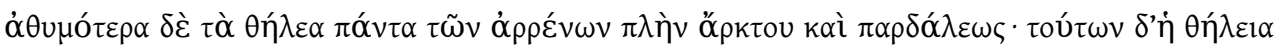

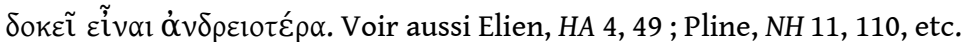

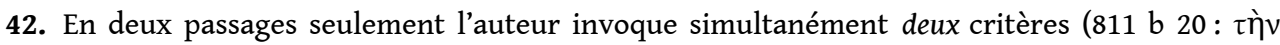

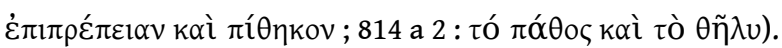

43. En particulier dans le traité hippocratique Airs, eaux et lieux 5 sq. (voir Foerster 1893, II, 241 sq.), comme le rappelle Galien (quod animi mores 4, 798-805).

44. Il est peut-être le concept clé de ce que la première partie du traité présente comme une voie originale de physiognomonie (807 a 4) et qui est la solidarité rationnelle de certains traits toujours associés dans les natures.

45. Les transcriptions modernes sont diverses et la question mériterait une analyse approfondie: «Gesamteindruck» (Vogt 1999: 403-405), «general appearance » (Mac c. Armstrong 1958 : 53), « evidenzza » (Raina 1993), « appropriate » (Hett 1936), etc.

46. Il pourrait s'agir de la correspondance typique intuitive entre le somatique et le psychique. Voir à ce propos Schmidt (1941: 1072) : «Den Deutungsversuchen der Physiognomiker liegt (zumeisst wohl unbewisst) ein Idealbild von der menschlichen gestalt und dem (im Idealfalle mit dem Ausseren in Harmonie übereinstimmenden) Wesensgehalt zugrunde ». Plutôt qu'une "harmonie intrinsèque des traits" (Laurand 2006) elle exprimerait un rapport avec un type humain parfait (voir e.g. Phgn. 810 b 31 : « les gens qui ont le dos rond ont une nature mauvaise ; on réfère cela à l'épiprepeia, parce que les parties antérieures, qui devraient être apparentes sont dissimulées »).

47. L'évaluation du sens de l' óv $\alpha \varphi \rho \rho \alpha ́$ dans le discours physiognomonique est complexe. L'analyse de l'expression, employée 114 fois dans la seconde partie des Physiognomonica aristotéliciens, est souvent esquivée par les critiques (e.g. Vogt 1999 : 418). 
48. Les traductions de la formule sont floues ou variables si rimanda ou si veda (Raina 1993), siehe (Vogt 1999), c'est le type de (André 1981, pour refertur ad dans AnL). Hett (1936) traduit diversement selon les cas, en donnant une valeur plus ou moins forte à la relation, alors que

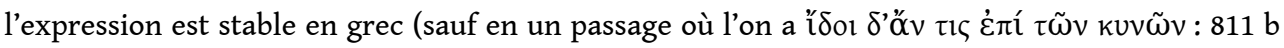
38) : this is due to (812 a 3), this refers to ( 811 b 4), this applies to (811 b 36), witness ( 810 b 32). La valeur causale accordée par Hett, surtout pour les renvois au critère pathotypique, s'explique par le fait que dans la majorité des cas (12 sur 17), l'indication est suivie d'une motivation introduite par ǒ $\imath$ ou contenant үó́ $\rho$. Mais ces précisions peuvent justifier la pertinence d'un rapprochement, sans faire du type invoqué une cause.

49. Voir infra 3.5 et 3.6.

50. ... sans compter des indications sur des zones anonymes du corps (base du nez) sa musculature générale et sa démarche.

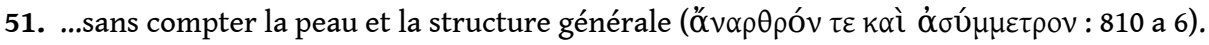

52. Sur l'extension large et imprécise du terme grec (panthère, léopard, guépard, lynx), voir Wotke 1949, qui signale que dans certains cas -surtout de $\pi \alpha ́ \alpha \delta \alpha \lambda \varepsilon ı \varsigma$ apprivoisées- « on peut identifier le guépard avec une quasi certitude» (p. 749). Mais curieusement le même auteur, traitant du portrait de cet animal dans la littérature physiognomonique oublie cette diversité et se contente de dire: "Eine Vorstellung jedoch, die von der Wircklichkeitsnähe der eben gekennzeichneten stark abweicht, finden wir bei der Physiognomikern» (p. 761).

53. La description d'Aristote est la suivante : petite face, large gueule, petits yeux plutôt pâles, enfoncés et très mobiles, front allongé, plutôt arrondi que plat au niveau des oreilles, cou extrêmement long et fin, poitrine étroite, dos long, croupe et cuisses charnues mais flancs et ventre plats ; couleur bariolée, ensemble désarticulé et mal proportionné (Phgn. $809 \mathrm{~b} 40$ sq.) ; celle dePolémon (26r, Foerster 1893 I, 196-7) s'y conforme : « panthera autem praedita est capite parvo fronte parva oculis acutis artubus mollibus collo angusto pectore arto lateribus gracilibus dorso magno, corpore levi crinibus mollibus». Le texte de Syllogé byzantine qui reprend l'Epitomé d'Aristophane de Byzance décrit de façon similaire le guépard, nommé $\pi$ óv $\theta \eta \rho$ dans ce texte (II, 279) : "Le guépard ressemble à la panthère ( $\left.\pi \alpha \alpha^{\alpha} \delta \alpha \lambda_{1 \varsigma}\right)$, à ceci près qu'il a un cou long

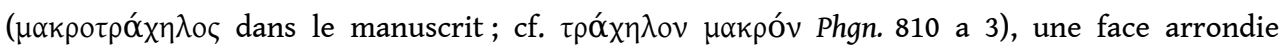

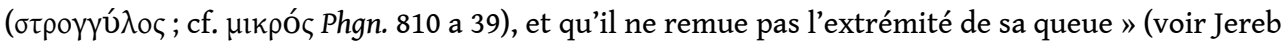
1949, $748:$ : $\pi \alpha ́ \alpha \theta \eta \eta$ und $\pi \alpha ́ \alpha \delta \alpha \lambda_{1 \varsigma}$ können als gleichbedeutend angenommen werden »).

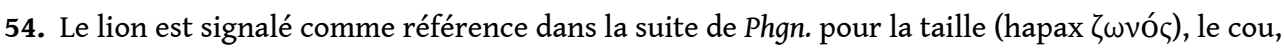
les lèvres, le nez, les yeux, le front, la couleur, la crinière, la pilosité, la démarche ; tandis que le guépard n'est plus signalé comme modèle pour une partie précise. Il y a donc un décalage entre le portrait somatique et l'utilisation du type dans le discours, et plusieurs régimes fonctionnels pour les figures animales.

55. Pour le détail des correspondance, voir Annexe 2.

56. Aristote, critiquant la pratique ordinaire des physiognomistes de tradition zoologique, indique nettement que les hommes ressemblent partiellement à un animal, relativement à un

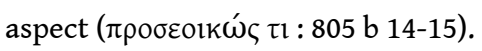

57. Ce principe, qui fait du diagnostic physiognomonique une opération géométrique et non arithmétique est affirmé par Aristote dans la conclusion du traité (814 a 3 ; voir Adamant. 1.3) et correspond au concept de dignitas signorum de l'Anonyme latin.

58. Voir AnL 132 : ex diversis animalibus de quibus iudicavimus signa conveniant in unum hominem.

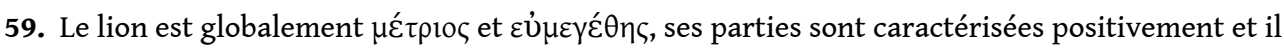

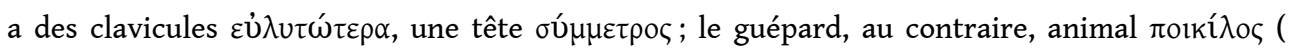

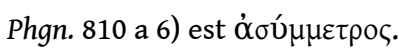

60. Voir Phgn. 811 a 36, 810 a 16, 810 a 25. Cf. aussi Phgn. 811 a 2 qui propose une formule presque tautologique : « ceux qui ont les épaules bien découplées ( $\varepsilon$ v̌ $\lambda v \tau o l)$ sont libres quant à leur esprit ; 


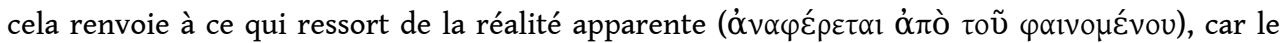

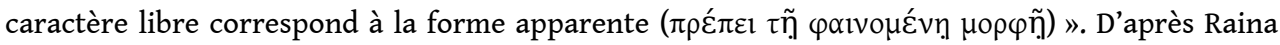

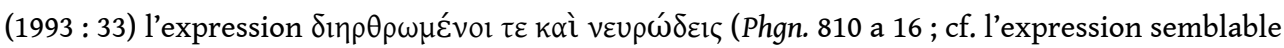
en 810 a 24) « è di sicura derivazione medica ».

61. Adamant. 2.1, Foerster 1893, I p. 348. Voir aussi en 2.2, Foerster 1893 , I p. 350 «Si chez un

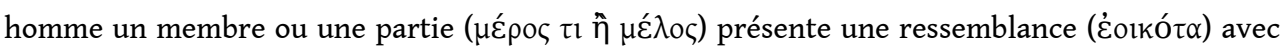
ceux d'une bête sauvage ou d'un oiseau, c'est à partir d'eux qu'il faut déterminer la nature

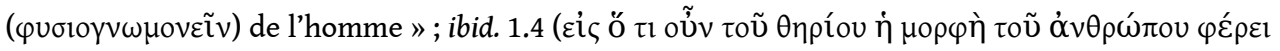

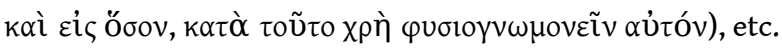

62. Le discours biologique pose que les êtres se distinguent essentiellement par les parties, et que cette différence est de trois types: nulle, quantitative, analogique; le discours physiognomonique adopte le même cadre et l'applique également à la mesure psychique, comme Aristote au livre VIII de NA (588 a 25 sq.; voir infra).

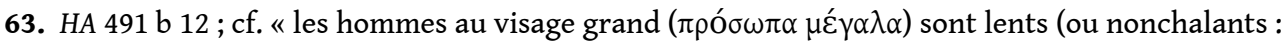
$v \omega ́ \theta \rho o \imath) »$; voir pour plus d'exemples de convergences Vogt 1999 : 133-145.

64. Voir PA II, chap. 2 \& 4, surtout 648 a 2 sq. ainsi que le problème 30, 1 (953 a 10 sq.) ; sur l'importance de la qualité du sang voir Lloyd $1983: 22$.

65. Notons au passage qu'Aristote indique que la liste des méthodes n'est pas close : «il existe

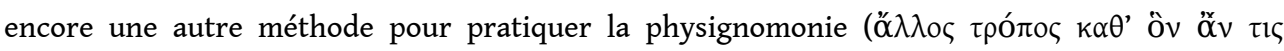

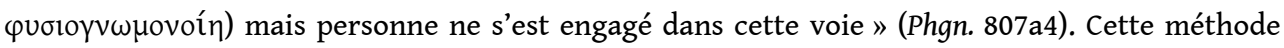
inédite, reposant sur le postulat du complexe caractériel, permettrait de déduire la présence d'un trait de caractère de la coprésence de traits toujours en connexion avec lui.

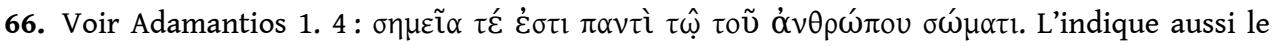
caractère intégral de la description du corps dans les sections anatomiques humaines des traités. 67. Pour la sélection des parties des douze animaux majeurs dans AnL voir Annexe 3.

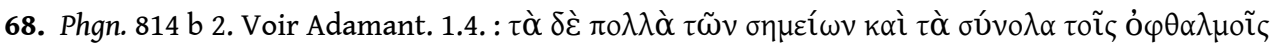

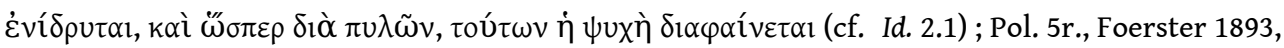
106 : scientiae physiognomoniae summa in oculi signis; AnL 45 : post oculos igitur vis signorum est quae juxta oculos et in vultu sunt, nam prout quaeque signa proxima sunt oculis, potiora sunt ceteris.

69. HA 491 b 9. Cf. Cicéron, Lois, 26 : « car les yeux extrêmement expressifs disent les émotions qui s'emparent de notre âme, et ce qu'on appelle le visage (vultus), qui ne peut exister chez aucun être vivant autre que l'homme, dénote le caractère (indicat mores) ».

70. Phgn. 811 b 4. Déjà Galien (Quod animi mores 4, 796), citant HA 491 b 1 hors contexte, semblait

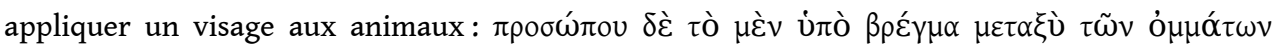

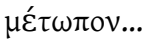

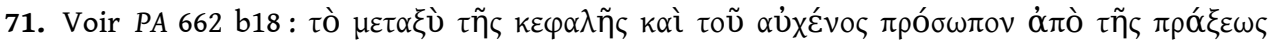

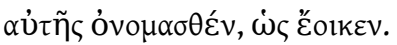

72. Voir Arist. HA 588 a 16 sq. Comme les traits des enfants, les traits animaux sont à la fois des

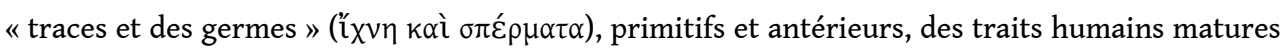
(cf. HA 608 b 4); sur cette question voir Zucker 2006.

73. Les équations «motivées" sont celles qui se réclament d'une analogie et ne se présentent pas comme des règles allant de soi.

74. Voir Lloyd 1983: 25. Ce caractère métaphorique de la vertu ou des qualités animales est

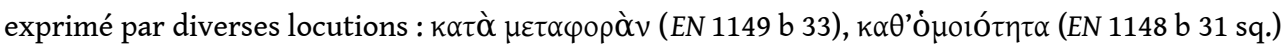

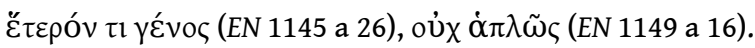




\section{RÉSUMÉS}

En Grèce, l'objet premier de la physiognomonie, dans sa forme ancienne et technique, est de manifester la correspondance entre sôma et psyché. Le rappel des principes et de la méthode de cette discipline permet de comprendre que l'enjeu est l'unité psycho-somatique (i.e. physique)des êtres vivants, attestée par la sympathie du corps et de l'esprit, et que ce discours est une somatologie avant d'être une psychologie. Pour déchiffrer l'identité psychique d'après les indices physiques le physiognomoniste recourt à des modèles de référence, dont les types animaux. L'étude, surtout dans le traité aristotélicien, de l'exploitation du corps animal et de la signification de ses différentes parties montre que l'interprétation analogique du psychisme de l'homme s'appuie sur un système de correspondances scientifique : l'anatomie comparée. Il n'y a nul délit $\mathrm{d}$ "anthropomorphisation' ici à constater l'identité structurelle fondamentale du corps animal et humain, comme de la psyché animale et humaine. Le défaut théorique de la physiognomonie est ailleurs : dans l'incapacité à formuler strictement et efficacement des règles de conversion entre traits psychiques et traits somatiques.

Ancient physiognomy and animal language of the bodyIn Greece, the main scope of the physiognomics, in its ancient and technical form, is to show the correspondence between body and mind. By reminding the principles and the method of this discipline we help to understand that the principal stake is the psychosomatic unity (i.e. physical) of living beings, demonstrated by the sympathy between body and mind, and that this speech is a somatology before being a psychology. To interpret the psychic identity according to the physical indications the physiognomist refers to models, among which the animal types. By studying, especially in the aristotelian treatise, the use of the animal body and the meaning attached to the various anatomical parts, it appears that the analogic interpretation of the human psychism is based on a scientific system of correspondences : the comparative anatomy. To admit the basic structural identity of the body by man and animal, as well as the identity of mind, is not an anthropomorphistic postulate. The theoretical flaw of physiognomics is not this, but in the fact that it is unable to express with rigour and relevance the rules of conversion between psychic and somatic features.

\section{INDEX}

Mots-clés : anatomie comparée, animal, Aristote, physiognomonie, psychosomatique, Psychosomatique, sympathie

Keywords : Adamantios, Animal, Aristotle, Comparative anatomy, Physiognomics, Psychosomatic

\section{AUTEUR}

\section{ARNAUD ZUCKER}

Université de Nice 